\title{
Higher K-theory of toric stacks
}

\author{
Roy Joshua AND Amalendu KRISHNA
}

\begin{abstract}
In this paper we develop several techniques for computing the higher G-theory and K-theory of quotient stacks. Our main results for computing these groups are in terms of spectral sequences. We show that these spectral sequences degenerate in the case of many toric stacks, thereby providing an efficient computation of their higher K-theory.

We apply our main results to give an explicit description for the higher Ktheory of many quotient stacks, including smooth toric stacks. We also show that our techniques apply to compute the higher K-theory of all spherical varieties over fields of characteristic 0 and all projective smooth spherical varieties over fields of arbitrary characteristics. As another application, we describe the higher $\mathrm{K}$-theory of toric stack bundles over smooth base schemes.
\end{abstract}

Mathematics Subject Classification (2010): 19L47 (primary); 14M25 (secondary).

\section{Introduction}

Toric varieties form a good testing ground for verifying many conjectures in algebraic geometry. This becomes particularly apparent when one wants to understand cohomology theories for algebraic varieties. Computations of cohomology rings of smooth toric varieties such as the Grothendieck ring of vector bundles, the Chow ring and the singular cohomology ring have been well-understood for many years. These computations facilitate predictions on the structure of various cohomology rings of a general algebraic variety.

Just like toric varieties, one would like to have a class of algebraic stacks on which various cohomological problems about stacks can be tested. The class of toric stacks, which can be studied in terms of combinatorial data called stacky fans, is precisely such a class of algebraic stacks.

These stacks are expected to be the toy models for understanding cohomology theories of algebraic stacks, a problem which is still very complicated in general.

The first author was supported by a grant from the National Science Foundation. The second author was supported by the Swarnajayanti fellowship, Govt. of India, 2011.

Received February 4, 2013; accepted in revised form November 25, 2013. 
Nevertheless, almost nothing has been worked out till now regarding the higher K-groups of toric stacks, even when they are Deligne-Mumford stacks, though the higher K-groups of toric varieties had been well understood ( $c f$. [41]) and the higher Chow groups of toric varieties have been computed recently in [23]. Furthermore, we still do not know how to compute even the Grothendieck K-theory ring of a general smooth toric stack.

In this paper we develop general techniques for computing the (integral) higher K-theory of smooth toric stacks, the main results being Theorems 1.1, 1.2 and 1.3. In fact, our results apply to a much bigger class of stacks than just toric stacks. For example, these results can be used to describe the higher equivariant K-theory of all projective smooth spherical varieties in any characteristic and all spherical varieties over a field of characteristic 0 . Our general results are in terms of spectral sequences as in (1.1) which we show degenerate in various cases of interest. This allows us to give explicit description of the higher K-theory of toric stacks.

As a consequence of this degeneration of spectral sequences, we recover (the integral versions of) and generalize all the previously known computations of the Grothendieck group of toric Deligne-Mumford stacks. We show how our main results can be used to describe the equivariant K-theory of spherical varieties. As further applications of the main results, we completely describe the (integral) higher K-theory of weighted projective spaces. As another application, we give a complete description of the higher K-theory of toric stack bundles over a smooth base scheme.

\subsection{Overview of the main results}

The following is an overview of our main results. We shall fix a base field $k$ throughout this text. A scheme in this paper will mean a separated and reduced scheme of finite type over $k$. A linear algebraic group $G$ over $k$ will mean a smooth and affine group scheme over $k$. By a closed subgroup $H$ of an algebraic group $G$, we shall mean a morphism $H \rightarrow G$ of algebraic groups over $k$ which is a closed immersion of $k$-schemes. In particular, a closed subgroup of a linear algebraic group will be of the same type and hence smooth. An algebraic group $G$ will be called diagonalizable if it is a product of a split torus over $k$ and a finite abelian group of order prime to the characteristic of $k$. In particular, we shall be dealing with only those tori which are split over $k$. Unless mentioned otherwise, all products of schemes will be taken over $k$. A $G$-scheme will mean a scheme with an action of the algebraic group $G$.

For a $G$-scheme $X$, let $\mathbf{G}^{G}(X)$ (respectively $\mathbf{K}^{G}(X)$ ) denote the spectrum of the K-theory of $G$-equivariant coherent sheaves (respectively vector bundles) on $X$. Let $R(G)$ denote the representation ring of $G$. This is canonically identified with $\mathbf{K}_{0}^{G}(k)$. If $\mathfrak{X}$ denotes an algebraic stack, we let $\mathbf{K}(\mathfrak{X})(\mathbf{G}(\mathfrak{X}))$ denote the Quillen K-theory (G-theory) of the exact category of vector bundles (coherent sheaves, respectively) on the stack $\mathfrak{X}$. For a quotient stack $\mathfrak{X}=[X / G]$, the spectrum $\mathbf{K}(\mathfrak{X})$ (respectively $\mathbf{G}(\mathfrak{X})$ ) is canonically weakly equivalent to the equivariant K-theory $\mathbf{K}^{G}(X)$ (respectively G-theory $\mathbf{G}^{G}(X)$ ) of $X$. See Section 2.3 for more details. 
Recall (see below) that a stacky toric stack $\mathfrak{X}$ is of the form $[X / G]$ where $X$ is a toric variety with dense torus $T$ and $G$ is a diagonalizable group acting on $X$ via a given morphism $\phi: G \rightarrow T$ of algebraic groups.

Our first result is the construction of a spectral sequence which allows one to compute the higher K-theory of the stack $[X / G]$ from the K-theory of the stack $[X / T]$, whenever a torus $T$ acts on a scheme $X$ and $\phi: G \rightarrow T$ is a morphism of diagonalizable groups. This is related to the spectral sequence in ( [27, Theorem $5.3]$ ), whose $E_{2}$-terms are expressed in terms of $\mathbf{G}_{*}([X / T])$ and which converges to the ordinary G-theory $\mathbf{G}_{*}(X)$. Merkurjev's construction is based on a similar construction of Levine [24].

Our spectral sequence is a generalization of [27, Theorem 5.3] in that the $E_{2}$ terms are given in terms of the $T$-equivariant $\mathrm{G}$-theory of a $T$-scheme and converge to the equivariant G-theory, equivariant with respect to a diagonalizable group mapping into the torus. This generalization allows one to write down the expression for the higher G-theory of all toric stacks. This is one of the main results of this paper. Furthermore, our spectral sequence can be used to compute the higher equivariant G-theory of all spherical varieties in characteristic zero and smooth projective spherical varieties in positive characteristic. In contrast to the spectral sequence of [27], our construction is much more elementary and is only based on a systematic study of the equivariant G-theory of linear varieties.

We also prove the degeneration of our spectral sequences in many cases, which provides an efficient tool for computing the higher K-theory of many quotient stacks, including toric stacks.

Theorem 1.1. Let $T$ be a split torus acting on a scheme $X$ and let $\phi: G \rightarrow T$ be a morphism of diagonalizable groups so that $G$ acts on $X$ via $\phi$. Then, there is a spectral sequence:

$$
E_{2}^{s, t}=\operatorname{Tor}_{s}^{R(T)}\left(R(G), \mathbf{G}_{t}([X / T])\right) \Rightarrow \mathbf{G}_{s+t}([X / G]) .
$$

Moreover, the edge map $\mathbf{G}_{0}([X / T]) \underset{R(T)}{\otimes} R(G) \rightarrow \mathbf{G}_{0}([X / G])$ is an isomorphism.

The spectral sequence (1.1) degenerates at the $E_{2}$-terms if $X$ is a smooth toric variety with dense torus $T$ such that $\mathbf{K}_{0}([X / T])$ is a projective $R(T)$-module and we obtain the ring isomorphism:

$$
\mathbf{K}_{*}([X / T]) \underset{R(T)}{\otimes} R(G) \stackrel{\simeq}{\rightarrow} \mathbf{K}_{*}([X / G]) .
$$

In particular, this isomorphism holds when $X$ is a smooth and projective toric variety with dense torus being $T$.

Observe that stackiness is allowed since the map $\phi: G \rightarrow T$ is not required to be injective. This makes the construction of the above spectral sequence and the identification of its $E_{2}$-terms rather surprising.

If $\mathfrak{X}=[X / G]$ is a generically stacky toric stack associated to the data $\underline{X}=$ $(X, G \stackrel{\phi}{\rightarrow} T)$, then the above results apply to the G-theory and K-theory of $\mathfrak{X}$. We 
shall apply Theorem 1.1 in Subsection 4.1 to give an explicit presentation of the Grothendieck K-theory ring of a smooth toric stack. If we specialize to the case of smooth toric Deligne-Mumford stacks, this recovers the main result of [4].

Another useful application of Theorem 1.1 is that it tells us how we can read off the $T^{\prime}$-equivariant G-groups of a $T$-scheme $X$ in terms of its $T$-equivariant Ggroups, whenever $T^{\prime}$ is a closed subgroup of $T$. The special case of the isomorphism $\mathbf{G}_{0}([X / T]) \underset{R(T)}{\otimes} R(G) \stackrel{\simeq}{\rightarrow} \mathbf{G}_{0}([X / G])$ when $G$ is the trivial group and $X$ is a smooth toric variety, recovers the main result of [28].

We should also observe that Theorem 1.1 applies to a bigger class of schemes than just toric varieties. In particular, one can use them to compute the equivariant $K$-theory of many spherical varieties. Another special case of the isomorphism $\mathbf{G}_{0}([X / T]) \underset{R(T)}{\otimes} R(G) \stackrel{\simeq}{\rightarrow} \mathbf{G}_{0}([X / G])$ when $G$ is the trivial group and $X$ is a spherical variety, recovers the main result of [35].

Theorem 1.2. Let $T$ be a split torus acting on a smooth and projective scheme $X$ which is T-equivariantly linear (cf. Definition 3.1). Let $\phi: G \rightarrow T$ be a morphism of diagonalizable groups so that $G$ acts on $X$ via $\phi$. Then the map

$$
\rho: \mathbf{K}_{0}([X / G]) \underset{\mathbb{Z}}{\otimes \mathbf{K}_{*}}(k) \simeq \mathbf{K}_{0}([X / G]) \underset{R(G)}{\otimes} \mathbf{K}_{*}^{G}(k) \rightarrow \mathbf{K}_{*}([X / G])
$$

is a ring isomorphism.

When $X$ is a smooth projective toric variety and $G=T$, then the above theorem recovers a result of Vezzosi-Vistoli [41, Theorem 6.9].

More generally, it turns out that Theorem 1.2 applies to all smooth projective spherical varieties. See Section 3 for related computations on the G-theory of equivariantly $T$-linear (not necessarily projective) schemes which, we show, includes all spherical varieties over fields of characteristic 0 (Corollary 3.7).

As an illustration of how the spectral sequence (1.1) degenerates in the cases not covered by Theorems 1.1 and 1.2, we prove the following result which describes the higher K-theory of toric stack bundles. As an intermediate step, we obtain a stacky version of the very useful Leray-Hirsch Theorem.

Theorem 1.3. Let $B$ be a smooth scheme over a perfect field $k$ and let $[X / G]$ be a toric stack where $X$ is smooth and projective. Let $R_{G}\left(\mathbf{K}_{*}(B), \Delta\right)$ denote the Stanley-Reisner algebra ( $c f$. Definition 7.1) over $\mathbf{K}_{*}(B)$ associated to a closed subgroup $G$ of $T$. Let $\pi: \mathfrak{X} \rightarrow B$ be a toric stack bundle with fiber $[X / G]$. Then there is a ring isomorphism

$$
\Phi_{G}: R_{G}\left(\mathbf{K}_{*}(B), \Delta\right) \stackrel{\simeq}{\rightarrow} \mathbf{K}_{*}(\mathfrak{X}) .
$$

When $G$ is the trivial group, the Grothendieck group $\mathbf{K}_{0}(\mathfrak{X})$, was computed in [32, Theorem 1.2(iii)]. When $[X / G]$ is a Deligne-Mumford stack, a computation of $\mathbf{K}_{0}(\mathfrak{X})$ appears in [19]. 
The paper [21] will continue the study begun here, by discussing the motivic cohomology (higher Chow groups) of toric stacks.

Here is an outline of the paper. The second section is a review of toric stacks and their K-theory. In Section 3, we define the notion of equivariantly linear schemes and study their G-theory. We prove Theorem 1.1 in Section 4, which is the most general result of this paper. We conclude this section with a detailed description of the Grothendieck K-theory ring of general smooth toric stacks.

In Section 5, we prove a derived Künneth formula and prove Theorem 1.2 as a consequence. We conclude this section by working out the higher K-theory of (stacky) weighted projective spaces. We study the K-theory of toric stack bundles over smooth base schemes in the last two sections and conclude by providing a complete determination of these.

ACKNOWLEDGEMENTS. Parts of this work were carried out while the first author was visiting the Tata Institute of Fundamental Research, while the second author was visiting the Mathematics department of Ohio state university, Columbus and also while both the authors were visiting the Mathematics department of the Harish Chandra Research Institute, Allahabad. The first author was also supported by an adjunct professorship at the same institute. They would would like to thank these departments for the invitation and financial support during these visits. They also would like to thank Michel Brion and Hsian-Hua Tseng for helpful comments on an earlier version of this paper.

\section{A review of toric stacks and their K-theory}

In this section, we review the concept of toric stacks from [14] and set up the notations for the G-theory and K-theory of such stacks. This is done in some detail for the convenience of the reader.

In what follows, we shall fix a base field $k$ and all schemes and algebraic groups will be defined over $k$. Let $\mathcal{V}_{k}$ denote the category of $k$-schemes and let $\mathcal{V}_{k}^{S}$ denote the full subcategory of smooth $k$-schemes. If $G$ is an algebraic group over $k$, we shall denote the category of $G$-schemes with $G$-equivariant maps by $\mathcal{V}_{G}$. The full subcategory of smooth $G$-schemes will be denoted by $\mathcal{V}_{G}^{S}$.

\subsection{Toric stacks}

Definition 2.1. Let $T$ be a torus and let $X$ be a toric variety with dense torus $T$. According to [14], a toric stack $\mathfrak{X}$ is an Artin stack of the form $[X / G]$ where $G$ is a subgroup of $T$.

A generically stacky toric stack is an Artin stack of the form $[X / G]$ where $G$ is a diagonalizable group with a morphism $\phi: G \rightarrow T$. In this case, the stack $\mathfrak{X}$ has an open substack of the form $[T / G]$ which acts on it. The action of $\mathfrak{T}=[T / G]$ 
on $\mathfrak{X}$ is induced from the torus action on $X$. The stack $\mathfrak{T}$ is often called the stacky dense torus of $\mathfrak{X}$. A generically stacky toric stack $[X / G]$ as above will often be described by the data $\underline{X}=(X, G \stackrel{\phi}{\rightarrow} T)$.

Examples 2.2. Generically stacky toric stacks arise naturally while one studies toric stacks. This is because a toric variety $X$ with dense torus $T$ has many $T$ invariant subvarieties which are toric varieties and whose dense tori are quotients of $T$. If $Z \subsetneq X$ is such a subvariety, and $G$ is a diagonalizable subgroup of the torus $T$, then $[Z / G]$ is not a toric stack but only a generically stacky toric stack.

A (generically stacky) toric stack $\mathfrak{X}$ is called a toric Deligne-Mumford stack if it is a Deligne-Mumford stack after forgetting the toric structure. It is called smooth if $X$ is a smooth scheme.

One extreme case of a toric stack is when $G$ is the trivial group, in which case $\mathfrak{X}$ is just a toric variety. The other extreme case is when $G$ is all of $T$ : clearly such toric stacks are Artin. In general, a toric stack occupies a place between these two extreme cases. If $\mathfrak{X}$ is a toric Deligne-Mumford stack, then the stacky torus $\mathfrak{T}$ is of the form $T^{\prime} \times \mathfrak{B}_{\mu}$, where $T^{\prime}$ is a torus and $\mathfrak{B}_{\mu}$ is the classifying stack of a finite abelian group $\mu$.

In general, every generically stacky toric stack can be written in the form $\mathfrak{X} \times$ $\mathfrak{B}_{G}$, where $\mathfrak{X}$ is a toric stack and $\mathfrak{B}_{G}$ is the classifying stack of a diagonalizable group $G$. This decomposition often reduces the study of the cohomology theories of generically stacky toric stacks to the study the cohomology theories of toric stacks and the classifying stacks of diagonalizable groups.

The coarse moduli space $\pi: \mathfrak{X} \rightarrow \bar{X}$ of a Deligne-Mumford toric stack is a simplicial toric variety whose dense torus is the moduli space of $\mathfrak{T}$. Conversely, every simplicial toric variety is the coarse moduli space of a canonically defined toric Deligne-Mumford stack ( $c f$. [11, Section 4.2]).

\subsection{Toric stacks via stacky fans}

In [14], Geraschenko and Satriano showed that all (generically stacky) toric stacks are obtained from stacky fans in much the same way toric varieties are obtained from fans. They describe in detail the dictionary between toric stacks and stacky fans.

Associated to the toric variety $X$ is a fan $\Sigma$ on the lattice of 1-parameter subgroups of $T, L=\operatorname{Hom}_{\mathrm{gp}}\left(\mathbb{G}_{m}, T\right)$ (see [12, Section 1.4] or [8, Section 3.1]). The surjection of tori $T \rightarrow T / G$ corresponds to the homomorphism of lattices of 1parameter subgroups, $\beta: L \rightarrow N=\operatorname{Hom}_{\mathrm{gp}}\left(\mathbb{G}_{m}, T / G\right)$. The dual homomorphism, $\beta^{*}: \operatorname{hom}(N, \mathbb{Z}) \rightarrow \operatorname{hom}(L, \mathbb{Z})$, is the induced homomorphism of characters. Since $T \rightarrow T / G$ is surjective, $\beta^{*}$ is injective, and the image of $\beta$ has finite index. Therefore, one may define a stacky fan as a pair $(\Sigma, \beta)$, where $\Sigma$ is a fan on a lattice $L$, and $\beta: L \rightarrow N$ is a homomorphism to a lattice $N$ such that $\beta(L)$ has finite index in $N$. Conversely, any stacky fan $(\Sigma, \beta)$ gives rise to a toric stack as follows.

Let $X_{\Sigma}$ be the toric variety associated to $\Sigma$. The dual of $\beta, \beta^{*}: N^{\vee} \rightarrow L^{\vee}$, induces a homomorphism of tori $T_{\beta}: T_{L} \rightarrow T_{N}$, naturally identifying $\beta$ with the 
induced map on lattices of 1-parameter subgroups. Since $\beta(L)$ is of finite index in $N, \beta^{*}$ is injective, so $T_{\beta}$ is surjective. Let $G_{\beta}=\operatorname{ker}\left(T_{\beta}\right)$. Note that $T_{L}$ is the torus of $X_{\Sigma}$ and $G_{\beta} \subseteq T_{L}$ is a subgroup. If $(\Sigma, \beta)$ is a stacky fan, the associated toric stack $\mathfrak{X}_{\Sigma, \beta}$ is defined to be $\left[X_{\Sigma} / G_{\beta}\right]$, with the torus $T_{N}=T_{L} / G_{\beta}$.

A generically stacky fan is a pair $(\Sigma, \beta)$, where $\Sigma$ is a fan on a lattice $L$, and $\beta: L \rightarrow N$ is a homomorphism to a finitely generated abelian group. If $(\Sigma, \beta)$ is a generically stacky fan, the associated generically stacky toric stack $\mathfrak{X}_{\Sigma, \beta}$ is defined to be $\left[X_{\Sigma} / G_{\beta}\right]$, where the action of $G_{\beta}$ on $X_{\Sigma}$ is induced by the homomorphism $G_{\beta} \rightarrow D\left(L^{*}\right)=T_{L}$.

One can give a more explicit description of $\mathfrak{X}_{\Sigma, \beta}$ considered above which will show that it is a generically stacky toric stack. Let $(\Sigma, \beta: L \rightarrow N)$ be a generically stacky fan and let $C(\beta)$ denote the complex $L \stackrel{\beta}{\rightarrow} N$. Let

$$
\mathbb{Z}^{s} \stackrel{Q}{\rightarrow} \mathbb{Z}^{r} \rightarrow N \rightarrow 0
$$

be a presentation of $N$, and let $B: L \rightarrow \mathbb{Z}^{r}$ be a lift of $\beta$ (which exists). One defines the fan $\Sigma^{\prime}$ on $L \oplus \mathbb{Z}^{s}$ as follows. Let $\tau$ be the cone generated by $e_{1}, \ldots, e_{s} \in \mathbb{Z}^{s}$. For each $\sigma \in \Sigma$, let $\sigma^{\prime}$ be the cone spanned by $\sigma$ and $\tau$ in $L \oplus \mathbb{Z}^{s}$. Let $\Sigma^{\prime}$ be the fan generated by all the $\sigma^{\prime}$. Corresponding to the cone $\tau$, we have the closed subvariety $Y \subseteq X_{\Sigma^{\prime}}$, which is isomorphic to $X_{\Sigma}$ since $\Sigma$ is the star (sometimes called the link) of $\tau$ [8, Proposition 3.2.7]. One defines

$$
\begin{aligned}
\beta^{\prime}=B \oplus Q: L \oplus \mathbb{Z}^{s} \longrightarrow \mathbb{Z}^{r} \\
(l, a) \longmapsto B(l)+Q(a) .
\end{aligned}
$$

Then $\left(\Sigma^{\prime}, \beta^{\prime}\right)$ is a generically stacky fan and we see that $\mathfrak{X}_{\Sigma, \beta} \simeq\left[Y / G_{\beta^{\prime}}\right]$. Note that $C\left(\beta^{\prime}\right)$ is quasi-isomorphic to $C(\beta)$, so $G_{\beta^{\prime}} \simeq G_{\beta}$.

Toric stacks and generically stacky toric stacks arise naturally, especially in the solution of certain moduli problems. Any toric variety naturally gives rise to a toric stack. In fact, it is shown in [15, Theorem 6.1] that if $k$ is algebraically closed field of characteristic zero, then every Artin stack with a dense open torus substack is a toric stack under certain fairly general conditions. We refer the readers to [14] where many examples of toric and generically stacky toric stacks are discussed.

In the rest of this paper, a toric stack will always mean any generically stacky toric stack. A toric stack as in Definition 2.1 will be called a reduced toric stack or a toric orbifold.

\subsection{K-theory of quotient stacks}

Let $G$ be a linear algebraic group acting on a scheme $X$. The spectrum of the $\mathrm{K}$-theory of $G$-equivariant coherent sheaves (respectively vector bundles) on $X$ is denoted by $\mathbf{G}^{G}(X)$ (respectively $\mathbf{K}^{G}(X)$ ). We will let $\mathbf{K}^{G}$ denote $\mathbf{K}^{G}$ (Spec $k$ ). The direct sum of the homotopy groups of these spectra are denoted by $\mathbf{G}_{*}^{G}(X)$ and $\mathbf{K}_{*}^{G}(X)$. The latter is a graded ring.

The natural map $\mathbf{K}^{G}(X) \rightarrow \mathbf{G}^{G}(X)$ is a weak equivalence if $X$ is smooth. 
This follows from [31, Theorem 3, Corollary 1.1]. In view of this, $\mathbf{G}^{G}(X)$ may be viewed as a ring spectrum when $X$ is smooth.

For a quotient stack $\mathfrak{X}$ of the form $[X / G]$, one writes $\mathbf{K}^{G}(X)$ and $\mathbf{K}(\mathfrak{X})$ interchangeably. The ring $\mathbf{K}_{0}^{G}(k)$ will be denoted by $R(G)$. This is same as the representation ring of $G$.

The functor $X \mapsto \mathbf{G}^{G}(X)$ on $\mathcal{V}_{G}$ is covariant for proper maps and contravariant for flat maps. It also satisfies the localization sequence and the projection formula. It satisfies the homotopy invariance property in the sense that if $f: V \rightarrow X$ is a $G$-equivariant vector bundle, then the map $f^{*}: \mathbf{G}^{G}(X) \rightarrow \mathbf{G}^{G}(V)$ is a weak equivalence. The functor $X \mapsto \mathbf{K}^{G}(X)$ on $\mathcal{V}_{G}$ is a contravariant functor with values in commutative graded rings. For any $G$-equivariant morphism $f: X \rightarrow Y, \mathbf{G}^{G}(X)$ is a module spectrum over the ring spectrum $\mathbf{K}^{G}(Y)$. In particular, $\mathbf{G}_{*}^{G}(X)$ is an $R(G)$-module. We refer to [36, Section 1] to verify the above properties.

\section{Equivariant G-theory of linear schemes}

We will prove Theorem 1.1 as a consequence of a more general result (Theorem 4.1) on the equivariant G-theory of schemes with a group action. In this section, we study the equivariant G-theory of a certain class of schemes which we call equivariantly linear. Such schemes in the non-equivariant set-up were earlier considered in [40]. The G-theory of such schemes in the non-equivariant set-up was studied in [20]. We end this section with a proof of Theorem 4.1 for equivariantly linear schemes.

Definition 3.1. Let $G$ be a linear algebraic group over $k$ and let $X \in \mathcal{V}_{G}$.

(1) We will say $X$ is $G$-equivariantly 0 -linear if it is either empty or isomorphic to $\operatorname{Spec}\left(\operatorname{Sym}\left(V^{*}\right)\right)$ where $V$ is a finite-dimensional rational representation of $G$.

(2) For a positive integer $n$, we will say that $X$ is $G$-equivariantly $n$-linear if there exists a family of objects $\{U, Y, Z\}$ in $\mathcal{V}_{G}$ such that $Z \subseteq Y$ is a $G$-invariant closed immersion with $U$ its complement, $Z$ and one of the schemes $U$ or $Y$ are $G$-equivariantly $(n-1)$-linear and $X$ is the other member of the family $\{U, Y, Z\}$.

(3) We will say that $X$ is $G$-equivariantly linear (or simply, $G$-linear) if it is $G$ equivariantly $n$-linear for some $n \geq 0$.

Definition 3.2. Let $G$ be a linear algebraic group over $k$. A scheme $X \in \mathcal{V}_{G}$ is called $G$-equivariantly cellular (or, $G$-cellular) if there is a filtration

$$
\emptyset=X_{n+1} \subsetneq X_{n} \subsetneq \cdots \subsetneq X_{1} \subsetneq X_{0}=X
$$

by $G$-invariant closed subschemes such that each $X_{i} \backslash X_{i+1}$ is isomorphic to a rational representation $V_{i}$ of $G$. These representations of $G$ are called the (affine) $G$-cells of $X$. 
It is immediate from the above definition that if $G \rightarrow G^{\prime}$ is a morphism of algebraic groups then every $G^{\prime}$-equivariantly linear scheme is also $G$-equivariantly linear. It is also obvious that a $G$-equivariantly cellular scheme is $G$-equivariantly linear.

Before we provide examples of equivariantly linear schemes, we state the following two elementary results which will be used throughout this paper.

Lemma 3.3. Let $G$ be a diagonalizable group over $k$ and let $H \subseteq G$ be a closed subgroup. Then $H$ is also defined over $k$ and is diagonalizable. If $T$ is a split torus over $k$, then all subtori and quotients of $T$ are defined over $k$ and are split over $k$.

Proof. The first statement follows from [3, Proposition 8.2]. If $T$ is a split torus over $k$, then any of its subgroups is defined over $k$ and is split by the first assertion. In particular, all quotients of $T$ are defined over $k$. Furthermore, all such quotients are split over $k$ by [3, Corollary 8.2].

Lemma 3.4. Let $T$ be a split torus acting on a reduced scheme $X$ with finitely many orbits. Then:

(1) Any $T$-orbit in X of minimal dimension is closed;

(2) Any $T$-orbit in $X$ of maximal dimension is open.

Proof. The first assertion is well known and can be found in [3, Proposition 1.8]. The second assertion is easily proved using (1) and an ascending induction on the number of $T$-orbits. The case where there is only one orbit is clear. In general, assume that $X$ has $n$ orbits and that (2) is true for $T$-schemes having up to $(n-1)$ orbits with $n \geq 2$.

Let $U \subseteq X$ be a $T$-orbit of maximal dimension. If all $T$-orbits have same dimension, then it follows from (1) that all orbits are closed and open. Otherwise, let us choose a $T$-orbit $Z$ of minimal dimension different from $U$. Then $Z \subsetneq X$ is closed by (1) and $X \backslash Z$ is a $T$-scheme with smaller number of $T$-orbits. Since $U \subseteq(X \backslash Z)$, it follows that $U$ is open in $X \backslash Z$ and hence in $X$.

Remark 3.5. The reader can verify that Lemma 3.4 is also true for the action of any diagonalizable group. But we do not need this general case.

The following results yield many examples of equivariantly linear schemes.

Proposition 3.6. Let $T$ be a split torus over $k$ and let $T^{\prime}$ be a quotient of $T$. Let $T$ act on $T^{\prime}$ via the quotient map. Then the following hold:

(1) $T^{\prime}$ is $T$-linear;

(2) A toric variety with dense torus $T$ is $T$-linear;

(3) A T-cellular scheme is T-linear;

(4) If $k$ is algebraically closed, then every $T$-scheme with finitely many $T$-orbits is T-linear. 
Proof. We first prove (1). It follows from Lemma 3.3 that $T^{\prime}$ is a split torus. Hence, it is enough to show using the remark following the definition of $T$-linear schemes that a split torus $T$ is $T$-linear under the multiplication action.

We can write $T=\left(\mathbb{G}_{m}\right)^{n}$ and consider $\mathbb{A}^{n}$ as the toric variety with the dense torus $T$ via the coordinate-wise multiplication so that the complement of $T$ is the union of the coordinate hyperplanes. Since $\mathbb{A}^{n}$ is $T$-linear, it suffices to show that the union of the coordinate hyperplanes is $T$-linear.

We shall prove by induction on the rank of $T$ that any union of the coordinate hyperplanes in $\mathbb{A}^{n}$ is $T$-linear. If $n=1$, then this is obvious. So let us assume that $n>1$ and let $Y$ be a union of some coordinate hyperplanes in $\mathbb{A}^{n}$. After permuting the coordinates, we can write $Y$ as $Y_{\{1, \cdots, m\}}^{n}=H_{1} \cup \cdots \cup H_{m}$ where $H_{i}=\left\{\left(x_{1}, \cdots, x_{n}\right) \in \mathbb{A}^{n} \mid x_{i}=0\right\}$. If $m=1$, then $Y_{\{1\}}^{n}$ is $T$-equivariantly 0-linear. So we assume by an induction on $m$ that $Y_{\{2, \cdots, m\}}^{n}$ is $T$-linear.

Set $U=Y_{\{1, \cdots, m\}}^{n} \backslash Y_{\{2, \cdots, m\}}^{n}$. Then $U$ is the complement of a union of hyperplanes $W_{\{2, \cdots, m\}}^{n-1}$ in $H_{1} \simeq \mathbb{A}^{n-1}$. Notice that $T$ acts on $H_{1}$ through the product $T_{1}$ of its last $(n-1)$ factors. By induction on $n$, we conclude that $W_{\{2, \cdots, m\}}^{n-1}$ is $T_{1}$-linear. Since $H_{1}$ is clearly $T_{1}$-linear, we conclude that $U$ is $T_{1}$-linear and hence $T$-linear. Thus we have concluded that both $Y_{\{2, \cdots, m\}}^{n}$ and $U$ are $T$-linear. It follows from this that $Y_{\{1, \cdots, m\}}^{n}$ is $T$-linear too.

The assertion (2) easily follows from (1) and an induction on the number of $T$-orbits in a toric variety. The assertion (3) is immediate from the definitions, using an induction on the length of the filtration of a $T$-cellular scheme. To prove (4), let $X$ be a $T$-scheme with only finitely many $T$-orbits. It follows from Lemma 3.4 that $X$ has an open $T$-orbit $U$. Since $k$ is algebraically closed, such an open $T$-orbit must be isomorphic to a quotient of $T$. In particular, it is $T$-linear by the first assertion. An induction of the number of $T$-orbit implies that $X \backslash U$ is $T$-linear. We conclude that $X$ is also $T$-linear.

\section{Spherical varieties}

Recall that if $G$ is a connected reductive group over $k$, then a normal variety $X \in \mathcal{V}_{G}$ is called spherical if a Borel subgroup of $G$ has a dense open orbit in $X$. The spherical varieties constitute a large class of varieties with group actions, including toric varieties, flag varieties and all symmetric varieties. It is known that a spherical variety $X$ has only finitely many fixed points for the $T$-action where $T$ is a maximal torus of $G$ contained in $B$.

It follows from a theorem of Bialynicki-Birula [2] (generalized to the case of non-algebraically closed fields by Hesselink [18]) that if $T$ is a split torus over $k$ and if $X$ is a smooth projective variety with a $T$-action such that the fixed point locus $X^{T}$ is isolated, then $X$ is $T$-equivariantly cellular. In this case, the filtration (3.1) gives rise to a $T$-equivariant stratification of $X$, known as the Bialynicki-Birula decomposition. We conclude that a smooth and projective spherical variety is $T$ cellular and hence $T$-linear. 
Using the Bialynicki-Birula decomposition and Proposition 3.6, we can now prove the following more general result in characteristic 0 . In particular, the results of the next two sections in this paper apply to all spherical varieties over fields of characteristic 0 .

Corollary 3.7. All spherical varieties over a field of characteristic 0 are $T$-linear.

Proof. The following argument was shown to us by Michel Brion [5]. Since every spherical variety has a stratification by finitely many $B$-orbits, it suffices to show that every $B$-orbit is $T$-equivariantly linear.

To prove this, one proceeds as follows. Let $\mathcal{O}$ be a fixed $B$-orbit, and let $G \mathcal{O}$ denote the $G$-orbit containing $\mathcal{O}$. Since we are in characteristic 0 , we can find a smooth toroidal compactification of $G \mathcal{O}$. Let us call this $Y$.

It follows from a result of Brion and Luna [6] that $\mathcal{O}$ is the intersection of $G \mathcal{O}$ with a $T$-cell of $Y$, coming from the above Bialynicki-Birula decomposition. Furthermore, this intersection is $T$-equivariantly isomorphic to $\mathbb{A}^{n} \backslash H$, where $H$ is a union some coordinate hyperplanes.

The $T$-representation $\mathbb{A}^{n}$ is $T$-linear and it follows from (the proof of) Proposition 3.6 that $H$ is $T$-linear. We conclude that $\mathcal{O}$ is $T$-linear.

\subsection{Equivariant G-theory of equivariantly linear schemes}

Recall that if a linear algebraic group $G$ acts on a scheme $X$, then the G-theory and $\mathrm{K}$-theory of the quotient stack $[X / G]$ are same as the equivariant G-theory and K-theory of $X$ for the action $G$. We shall use this identification throughout this text without further mention. The following result will be used repeatedly in this text.

Theorem 3.8. Let $G$ be a linear algebraic group over $k$ and let $H \subseteq G$ be a closed subgroup of $G$. Then for any $X \in \mathcal{V}_{H}$, the map of spectra

$$
\mathbf{G}\left(\left[\left({ }^{\stackrel{H}{\times}} G\right) / G\right]\right) \rightarrow \mathbf{G}([X / H])
$$

is a weak equivalence. In particular, the map of spectra

$$
\mathbf{G}([(X \times G / H) / G]) \rightarrow \mathbf{G}([X / H])
$$

is a weak equivalence if $X \in \mathcal{V}_{G}$. These are weak equivalences of ring spectra if $X$ is smooth.

Proof. Recall that the inclusion $X \times H \hookrightarrow X \times G$ induces a regular closed immersion $\iota: X \simeq X \stackrel{H}{\times} H \hookrightarrow X \stackrel{H}{\times} G$ which is $H$-equivariant, where $H$ acts on $X \stackrel{H}{\times} G$ by $h \star(x, g)=\left(x, g h^{-1}\right)$. In fact, this extends to a $G$-action on $X \stackrel{H}{\times} G$. We conclude that there are morphisms of stacks

$$
[X / H] \rightarrow[(X \stackrel{H}{\times} G) / H] \rightarrow(X \stackrel{H}{\times} G) / G]
$$


where the first map is a regular closed immersion and second map is smooth. In particular, there are pull-back maps of spectra

$$
\mathbf{G}([(X \stackrel{H}{\times} G) / G]) \rightarrow \mathbf{G}([X / H]) ; \mathbf{K}([(X \stackrel{H}{\times} G) / G]) \rightarrow \mathbf{K}([X / H]) .
$$

Since the second map is induced by pulling back vector bundles and preserves their tensor products, it is a morphism of ring spectra, as is well-known (see [39, Section 1.4]). It is shown in [38, Proposition 6.2] that the maps in (3.3) are weak equivalences. It follows that the second map (hence the first map as well if $X$ is smooth: see (2.1)) is a weak equivalence of ring spectra. Recall here that a weak equivalence of ring spectra means a morphism of ring spectra which is a weak equivalence after forgetting the ring structures.

Remark 3.9. We remark here that the weak equivalences of Theorem 3.8 are in fact natural in the category $\mathcal{V}_{H}$. This is because the morphisms in (3.2) are natural in $X \in \mathcal{V}_{H}$.

\subsubsection{Derived smash product of spectra}

Recall that for a stack $\mathfrak{X}$, the $\mathrm{K}$-theory spectrum $\mathbf{K}(\mathfrak{X})$ is a commutative ring spectrum and $\mathbf{G}(\mathfrak{X})$ is a module spectrum over $\mathbf{K}(\mathfrak{X})$. In the following results, we make essential use of the derived smash products of module spectra over ring spectra. This is the derived functor of the smash product of spectra in their homotopy category. We refer to [10, Chapter II, Section 3] (see also [33, Section 3] and [20, Section 3]) for notion of commutative ring spectra and their module spectra. The commutative ring spectra are also called $E_{\infty}$ rings in [10] .

For a commutative ring spectrum $R$, the category $\operatorname{Mod}_{R}$ of $R$-module spectra inherit a model structure, where the weak equivalence and cofibrations are the same as the (stable) weak equivalence and cofibrations of ordinary spectra (see [33, Theorem 4.1]). The associated homotopy category is denoted by $\mathcal{D}_{R}$. The smash product of $R$-module spectra induces a derived smash product functor

$$
(-) \stackrel{L}{\wedge}(-): \mathcal{D}_{R} \times \mathcal{D}_{R} \rightarrow \mathcal{D}_{R}
$$

For $M, N \in \operatorname{Mod}_{R}$, the derived smash product $M \stackrel{L}{\wedge} N$ is the class (in $\mathcal{D}_{R}$ ) of the smash product $C(M) \underset{R}{\wedge} N$, where $C(M) \rightarrow M$ is a cofibrant replacement of $M$ in $\operatorname{Mod}_{R}$. In fact, as is true with any model category, there is a functor $C(-)$ : $\operatorname{Mod}_{R} \rightarrow \operatorname{Mod}_{R}$ and a natural transformation $\tau: C \rightarrow$ Id such that $C(M) \rightarrow M$ is a cofibrant replacement for every $M \in \operatorname{Mod}_{R}$. The cofibrant replacement has the property that $C(M){ }_{R}^{\wedge}-$ preserves weak equivalences and cofibration sequences in the argument - . The cofibrant replacement $C(M) \rightarrow M$ coincides with a projective resolution in the abelian case (i.e., for chain complexes). 
We refer to [10, page 60] for the construction of the above derived smash product functor, where $M \stackrel{L}{\wedge} N$ is simply written as $M \underset{R}{\wedge} N$. In ibid., the cofibrant replacements $C(M)$ are explicitly constructed in terms of cell (free) $R$-modules (see [ibid., Chapter III, Section 2]).

The homotopy groups of the derived smash product $M_{R}^{\stackrel{L}{\wedge}} N$ is computed using the Eilenberg-Moore type spectral sequence:

$$
E_{s, t}^{2}=\operatorname{Tor}_{s, t}^{\pi_{*}(R)}\left(\pi_{*}(M), \pi_{*}(N)\right) \Rightarrow \pi_{s+t}(\stackrel{M}{\stackrel{L}{\wedge}} N) .
$$

This spectral sequence is obtained as a special case of [10, Chapter IV, (6.1)] by taking $E$ there to be the sphere spectrum.

We shall now prove the following special case of Theorem 4.1. The proof follows a trick used in [20, Theorem 4.1] in a different context. Let $G \rightarrow H$ denote a homomorphism of diagonalizable groups and let $H$ act on a scheme $X$. In the notation above, we let $R=\mathbf{K}^{H}, M=\mathbf{K}^{G}$ and $N=\mathbf{G}([X / H])$. This gives us maps in $\mathcal{D}_{R}$ :

$$
\mathbf{K}_{\mathbf{K}^{H}}^{\stackrel{L}{\wedge}} \mathbf{G}([X / H]) \stackrel{\tau}{\rightarrow} \mathbf{K}^{G} \underset{\mathbf{K}^{H}}{\wedge} \mathbf{G}([X / H]) \rightarrow \mathbf{G}([X / G]) .
$$

The functoriality of $\mathbf{K}^{G}{ }_{\mathbf{K}^{H}}^{\stackrel{L}{\wedge}}(-)$ and naturality of $\tau$ show that the first map is natural in $X$. The last map is clearly natural in $X$. (This map uses the pairing that sends a $G$ representation tensored with an $H$-equivariant coherent sheaf on $X$ to the obvious $G$-equivariant coherent sheaf on $X$.) We shall denote this composite map by $\tau_{X}^{G, H}$.

Proposition 3.10. Let $T$ be a split torus and let $X \in \mathcal{V}_{T}$ be T-linear. Let $\phi: G \rightarrow$ $T$ be a morphism of diagonalizable groups such that $G$ acts on $X$ via $\phi$. Then the natural map of spectra

$$
\tau_{X}^{G, T}: \mathbf{K}([\operatorname{Spec}(k) / G]) \underset{\mathbf{K}([\operatorname{Spec}(k) / T])}{\stackrel{L}{\wedge}} \mathbf{G}([X / T]) \rightarrow \mathbf{G}([X / G])
$$

is a weak equivalence.

Proof. We assume that $X$ is $T$-equivariantly $n$-linear for some $n \geq 0$. We shall prove our result by an ascending induction on $n$. If $n=0$, then $X \simeq \mathbb{A}^{n}$ and hence by the homotopy invariance, we can assume that $X=\operatorname{Spec}(k)$, and the result is immediate in this case. We now assume that $n>0$. By the definition of $T$-linearity, there are two cases to consider:

(1) There exists a $T$-invariant closed subscheme $Y$ of $X$ with complement $U$ such that $Y$ and $U$ are $T$-equivariantly $(n-1)$-linear. 
(2) There exists a $T$-scheme $Z$ which contains $X$ as a $T$-invariant open subscheme such that $Z$ and $Y=Z \backslash X$ are $T$-equivariantly $(n-1)$-linear.

In the first case, the localization fiber sequence in equivariant G-theory gives us a commutative diagram of fiber sequences in the homotopy category of spectra $^{1}$ : The left and the right vertical maps are weak equivalences by the induction. We

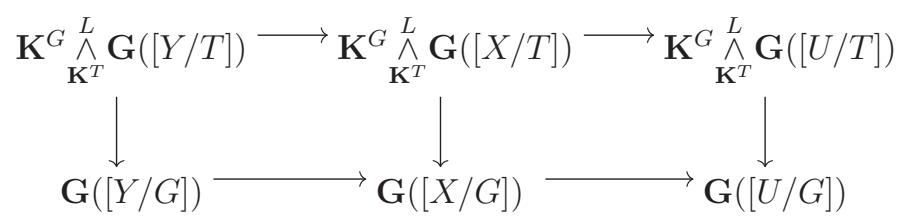

conclude that the middle vertical map is a weak equivalence too.

In the second case, we obtain as before, a commutative diagram of fiber sequences in the homotopy category of spectra:

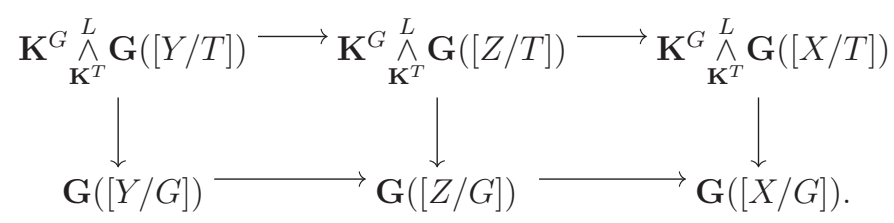

The first two vertical maps are weak equivalences by induction and hence the last vertical map must also be a weak equivalence. This completes the proof of the proposition.

Lemma 3.11 ([36, Lemma 5.6]). Let $G \rightarrow H$ be an epimorphism of diagonalizable groups and let $X$ be an affine scheme with an $H$-action. Then the following hold:

(1) The natural map at the level of homotopy groups

$$
R(G) \underset{R(H)}{\otimes} \mathbf{G}_{*}([X / H]) \rightarrow \mathbf{G}_{*}([X / G])
$$

is an isomorphism;

(2) The map of spectra

$$
\tau_{X}^{G, H}: \mathbf{K}_{\mathbf{K}^{H}}^{G} \stackrel{L}{\wedge} \mathbf{G}([X / H]) \rightarrow \mathbf{G}([X / G])
$$

is a weak equivalence.

Proof. Item (1) follows directly from [36, Lemma 5.6]. (In fact, though the notation in [36, Lemma 5.6] suggests a statement at the level of spectra, it is essentially a statement at the level of the homotopy groups of the equivariant G-theory spectra.)

${ }^{1}$ For spectra, this is same as a cofiber sequence 
One can deduce (2) using (1) and the spectral sequence (3.5) as follows and (2) may be viewed as the correct spectrum-level version of [36, Lemma 5.6].

We need to show that the map of spectra in (2) induces isomorphism between the homotopy groups. In view of (1), it suffices to show that the spectral sequence (3.5) degenerates and its $E^{2}$-terms are identified with $R(G) \underset{R(H)}{\otimes} \mathbf{G}_{*}([X / H])$.

However, the epimorphism $G \rightarrow H$ implies that the map of character groups $\widehat{H} \rightarrow \widehat{G}$ is a monomorphism of finitely generated abelian groups. Since the representation ring of a split diagonalizable group is the group ring of its characters, it follows from Lemma 3.12 that $R(G)$ is a free $R(H)$-module.

Using this and applying (1) for $X=\operatorname{Spec}(k)$, we see that $\mathbf{K}_{*}^{G}(k)$ is a free $\mathbf{K}_{*}^{H}(k)$-module. But this implies that (3.5) degenerates, identifying the homotopy groups on the left-hand-side with $R(G) \underset{R(H)}{\otimes} \mathbf{G}_{*}([X / H])$.

The following result should be known to experts. We provide a proof due to lack of a suitable reference.

Lemma 3.12. Let $M \hookrightarrow N$ be a monomorphism of finitely generated abelian groups. Let $S \subseteq N$ be a complete set of representatives of cosets of $M$ in $N$. Then $\mathbb{Z}[N]$ is a free $\mathbb{Z}[M]$-module with basis $S$.

Proof. Let $F$ denote the free $\mathbb{Z}[M]$-module generated by $S$. Let us define a $\mathbb{Z}[M]$ linear map

$$
\theta: F \rightarrow \mathbb{Z}[N] ; \quad \theta\left(\sum_{g \in S} x_{g} \star g\right)=\sum_{g \in S} x_{g}[g] .
$$

To show the surjectivity of $\theta$, let $x \in \mathbb{Z}[N]$. We can write $x=x_{1}+\cdots+x_{n}$, where $x_{i} \in \mathbb{Z}\left[s_{i} M\right]$. This gives us $x=\sum_{i=1}^{n}\left(x_{i}\left(s_{i}\right)^{-1}\right) s_{i}$ and $x_{i}\left(s_{i}\right)^{-1} \in \mathbb{Z}[M]$.

To show the injectivity of $\theta$, suppose that $\sum_{g \in S} x_{g}[g]=0$ in $\mathbb{Z}[N]$ and take any $h \in S$. Then we get $\sum_{g \in S} x_{g}[g]\left[h^{-1}\right]=0$. Since $g h^{-1} \in M$ if and only if $g=h$ (by choice of $S$ ), we see that the term on the left side of the last identity is simply $x_{h}$, which must thus be zero.

We end this section with the following (rather technical) result which will be used in the proof of Theorem 4.1. Taking $V$ to be $\operatorname{Spec}(k)$, this becomes a special case of what is considered in the last Proposition.

Lemma 3.13. Let $T$ be a split torus over $k$ and let $T^{\prime}$ be a quotient of $T$. Let $T$ act on $T^{\prime}$ via the quotient map and let it act trivially on an affine scheme $V$. Consider the scheme $X=V \times T^{\prime}$ where $T$ acts diagonally. Let $\phi: G \rightarrow T$ be a morphism of diagonalizable groups such that $G$ acts on any $T$-scheme via $\phi$. Then the map of spectra

$$
\tau_{X}^{G, T}: \mathbf{K}([\operatorname{Spec}(k) / G]) \underset{\mathbf{K}([\operatorname{Spec}(k) / T])}{\stackrel{L}{\wedge}} \mathbf{G}([X / T]) \rightarrow \mathbf{G}([X / G])
$$

is a weak equivalence. 
Proof. Let $H$ denote the image of $G$ in $T^{\prime}$ under the composite map $G \stackrel{\phi}{\rightarrow} T \rightarrow T^{\prime}$ and let $H^{\prime}=T^{\prime} / H$. Notice that $T^{\prime}$ is a split torus by Lemma 3.3. Since $T$ (and hence $G$ ) acts trivially on the scheme $V$, it follows that $T$ and $G$ act on $X$ via their quotients $T^{\prime}$ and $H$, respectively. Since $X$ is affine and all the underlying groups are diagonalizable, it follows from Lemma 3.11 that the maps of spectra

$$
\begin{gathered}
\mathbf{K}^{T} \underset{\mathbf{K}^{T^{\prime}}}{\stackrel{L}{\wedge} \mathbf{G}\left(\left[X / T^{\prime}\right]\right)} \rightarrow \mathbf{G}([X / T]) ; \\
\mathbf{K}^{G} \underset{\mathbf{K}^{H}}{\stackrel{L}{L} \mathbf{G}([X / H])} \rightarrow \mathbf{G}([X / G])
\end{gathered}
$$

are weak equivalences. Using the first weak equivalence, we obtain

$$
\begin{aligned}
& \mathbf{K}^{G} \underset{\mathbf{K}^{T}}{\stackrel{L}{\wedge}} \mathbf{G}([X / T]) \simeq \mathbf{K}^{G} \underset{\mathbf{K}^{T}}{\stackrel{L}{\wedge}}\left(\mathbf{K}^{T} \underset{\mathbf{K}^{T^{\prime}}}{\stackrel{L}{\wedge}} \mathbf{G}\left(\left[X / T^{\prime}\right]\right)\right) \\
& \simeq \mathbf{K}^{G} \underset{\mathbf{K}^{T^{\prime}}}{\stackrel{L}{\wedge}} \mathbf{G}\left(\left[X / T^{\prime}\right]\right) \\
& \simeq\left(\mathbf{K}^{G} \underset{\mathbf{K}^{H}}{\stackrel{L}{\wedge}} \mathbf{K}^{H}\right) \underset{\mathbf{K}^{T^{\prime}}}{\wedge} \mathbf{G}\left(\left[X / T^{\prime}\right]\right) \\
& \simeq \mathbf{K}^{G} \underset{\mathbf{K}^{H}}{\stackrel{L}{\wedge}}\left(\mathbf{K}^{H} \underset{\mathbf{K}^{T^{\prime}}}{\stackrel{L}{L}} \mathbf{G}\left(\left[X / T^{\prime}\right]\right)\right) .
\end{aligned}
$$

On the other hand, we have

$$
\begin{aligned}
\mathbf{K}^{H} \stackrel{\wedge_{\mathbf{K}^{T^{\prime}}}^{L} \mathbf{G}\left(\left[X / T^{\prime}\right]\right)}{ } & { }^{1} \mathbf{G}\left(\left[H^{\prime} / T^{\prime}\right]\right) \stackrel{L}{\wedge} \mathbf{G}\left(\left[X / T^{\prime}\right]\right) \\
& \simeq^{2} \mathbf{G}\left(\left[\left(H^{\prime} \times X\right) / T^{\prime}\right]\right) \\
& \simeq^{3} \mathbf{G}([X / H]),
\end{aligned}
$$

where the isomorphisms $\simeq^{1}$ and $\simeq^{3}$ follow from Theorem 3.8. The isomorphism $\simeq^{2}$ follows from Propositions 3.6 and 5.1. Combining (3.9), (3.10) and (3.11), we get the weak equivalences

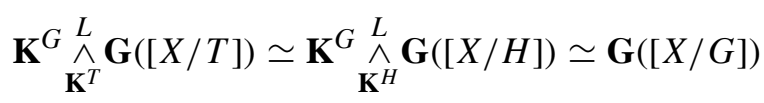

and this proves the lemma.

\section{G-theory of general toric stacks}

This section is devoted to the determination of the G-theory of a general (generically stacky) toric stack. We prove our main results in a much more general set-up where the underlying scheme with a $T$-action need not be a toric variety. 
Our first result is a spectral sequence that computes the $G$-equivariant G-theory of a $T$-scheme $X$ in terms of its $T$-equivariant G-theory and the representation ring of $G$ whenever there is a morphism of diagonalizable groups $\phi: G \rightarrow T$. When the underlying scheme is assumed to be smooth, these conclusions may be stated in terms of K-theory instead of G-theory.

This result specializes to the case of all (generically stacky) toric stacks when $X$ is assumed to be a toric variety. We conclude this section with an explicit presentation of the Grothendieck K-theory ring of a smooth toric stack which may not necessarily be Deligne-Mumford.

We now prove the following main result of this section and derive its consequences.

Theorem 4.1. Let $T$ be a split torus acting on a scheme $X$ and let $\phi: G \rightarrow T$ be a morphism of diagonalizable groups such that $G$ acts on $X$ via $\phi$. Then the natural map of spectra

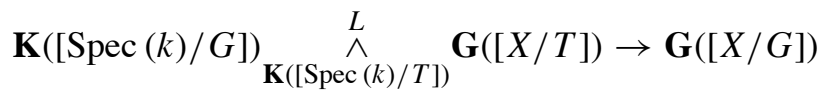

is a weak equivalence. In particular, one obtains a spectral sequence:

$$
E_{s, t}^{2}=\operatorname{Tor}_{s, t}^{\mathbf{K}_{*}^{T}(k)}\left(\mathbf{K}_{*}^{G}(k), \mathbf{G}_{*}([X / T])\right) \Rightarrow \mathbf{G}_{s+t}([X / G]) .
$$

Proof. We shall prove the theorem by the Noetherian induction on $T$-schemes. The statement of the theorem is obvious if $X$ is the empty scheme so that both sides of (4.1) are contractible. Suppose $X$ is any $T$-scheme such that (4.1) holds when $X$ is replaced by all its proper $T$-invariant closed subschemes. We show that (4.1) holds for $X$. This will prove the theorem.

By Thomason's generic slice theorem [37, Proposition 4.10], there exists a $T$ invariant dense open subset $U \subseteq X$ which is affine. Moreover, $T$ acts on $U$ via its quotient $T^{\prime}$ which in turn acts freely on $U$ with affine geometric quotient $U / T$ such that there is a $T$-equivariant isomorphism $U \simeq(U / T) \times T^{\prime}$. Here, $T$ acts trivially on $U / T$, via the quotient map on $T^{\prime}$ and diagonally on $U$. The weak equivalence of (4.1) holds for $U$ by Lemma 3.13.

We now set $Y=X \backslash U$. Then $Y$ is a proper $T$-invariant closed subscheme of $X$. The localization sequence induces the commutative diagram of the fiber sequences in the homotopy category of spectra:

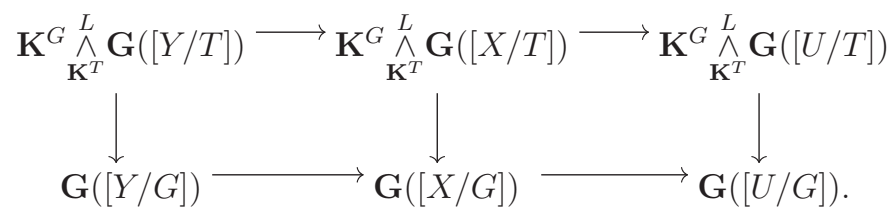

We have shown above that the right vertical map is a weak equivalence. The left vertical map is a weak equivalence by the Noetherian induction. We conclude that the middle vertical map is a weak equivalence too. The existence of the spectral sequence now follows from (3.5). 
Proof of Theorem 1.1. To obtain the spectral sequence (1.1), it is enough to identify this spectral sequence with the one in (4.2).

To see this, we recall from Lemma 3.11 that the maps

$$
R(T) \underset{\mathbb{Z}}{\otimes \mathbf{K}_{*}}(k) \rightarrow \mathbf{K}_{*}([\operatorname{Spec}(k) / T])
$$

and $R(G) \otimes \mathbf{K}_{*}(k) \rightarrow \mathbf{K}_{*}([\operatorname{Spec}(k) / G])$ are ring isomorphisms. Since $R(T)$ and $R(G)$ are flat $\mathbb{Z}$-modules, these isomorphisms can be written as

$$
\begin{aligned}
R(T) \underset{\mathbb{Z}}{\stackrel{L}{\otimes} \mathbf{K}_{*}(k)} & \stackrel{\simeq}{\rightarrow} \mathbf{K}_{*}([\operatorname{Spec}(k) / T]) \text { and } R(G) \underset{\mathbb{Z}}{\otimes} \mathbf{K}_{*}(k) \\
& \stackrel{\simeq}{\rightarrow} \mathbf{K}_{*}([\operatorname{Spec}(k) / G]),
\end{aligned}
$$

where $\stackrel{L}{\otimes}$ denotes the derived tensor product.

Let $M^{\bullet} \stackrel{\sim}{\rightarrow} R(G)$ be a flat resolution of $R(G)$ as an $R(T)$-module. Since $R(T)$ is a flat $\mathbb{Z}$-module, we see that $M^{\bullet} \stackrel{\sim}{\rightarrow} R(G)$ is a flat resolution of $R(G)$ also as a $\mathbb{Z}$-module. In particular, we obtain

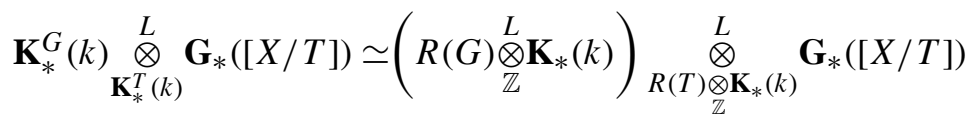

$$
\begin{aligned}
& \simeq\left(M \cdot \underset{\mathbb{Z}}{\otimes} \mathbf{K}_{*}^{L}(k)\right) \underset{R(T) \underset{\mathbb{Z}}{\otimes} \mathbf{K}_{*}(k)}{L} \mathbf{G}_{*}([X / T]) \\
& \simeq^{1}\left(M^{\bullet} \underset{\mathbb{Z}}{\otimes \mathbf{K}_{*}}(k)\right) \underset{R(T) \underset{\mathbb{Z}}{\otimes} \mathbf{K}_{*}(k)}{\stackrel{L}{\otimes}} \mathbf{G}_{*}([X / T]) \\
& \simeq^{2}\left(M^{\bullet} \underset{\mathbb{Z}}{\otimes \mathbf{K}_{*}}(k)\right) \underset{R(T) \underset{\mathbb{Z}}{\otimes} \mathbf{K}_{*}(k)}{\otimes} \mathbf{G}_{*}([X / T]) \\
& \simeq M^{\bullet} \underset{R(T)}{\otimes}\left(R(T) \underset{\mathbb{Z}}{\otimes \mathbf{K}_{*}}(k)\right) \underset{R(T) \underset{\mathbb{Z}}{\otimes} \mathbf{K}_{*}(k)}{\otimes} \mathbf{G}_{*}([X / T]) \\
& \simeq M^{\bullet} \otimes \mathbf{G}_{*}([X / T]) \\
& R(T) \\
& \simeq^{3} M^{\bullet} \stackrel{\bigotimes_{R(T)}^{L}}{\otimes} \mathbf{G}_{*}([X / T]) \\
& \simeq R(G) \underset{R(T)}{\otimes} \mathbf{G}_{*}([X / T]),
\end{aligned}
$$

where the isomorphism $\simeq^{1}$ follows because $M^{\bullet}$ is a complex of flat $\mathbb{Z}$-modules, $\simeq^{2}$ follows because $M^{\bullet} \underset{\mathbb{Z}}{\otimes \mathbf{K}_{*}}(k)$ is a complex of flat $R(T) \underset{\mathbb{Z}}{\otimes \mathbf{K}_{*}}(k)$-modules and the isomorphism $\simeq^{3}$ follows because $M^{\bullet}$ is a complex of flat $R(T)$-modules. Taking 
the homology groups on the both sides, we obtain

$$
\operatorname{Tor}_{s, t}^{\mathbf{K}_{*}^{T}(k)}\left(\mathbf{K}_{*}^{\mathbf{G}}(k), \mathbf{G}_{*}([X / T])\right) \simeq \operatorname{Tor}_{s, t}^{R(T)}\left(R(G), \mathbf{G}_{*}([X / T])\right)
$$

which yields the spectral sequence (1.1). The isomorphism of the edge map $\mathbf{G}_{0}([X / T]) \underset{R(T)}{\otimes} R(G) \rightarrow \mathbf{G}_{0}([X / G])$ follows immediately from (1.1) and the fact that the equivariant G-theory spectra appearing in Theorem 1.1 are all connective (have no negative homotopy groups).

Let us now assume that $X$ is a smooth toric variety with dense torus $T$ such that $\mathbf{K}_{0}([X / T])$ is a projective $R(T)$-module. In this case, we can identify the Gtheory and the K-theory. To show the degeneration of the spectral sequence (1.1), it suffices to show that the map

$$
R(G) \underset{R(T)}{\otimes} \mathbf{G}_{*}([X / T]) \rightarrow R(G) \underset{R(T)}{\otimes} \mathbf{G}_{*}([X / T])
$$

is an isomorphism. However, we have

$$
\begin{aligned}
& R(G) \underset{R(T)}{\otimes} \mathbf{G}_{*}([X / T]) \cong 0 \quad R(G) \underset{R(T)}{\otimes}\left(\mathbf{G}_{0}([X / T]) \underset{R(T)}{\otimes} \mathbf{K}_{*}^{T}(\operatorname{Spec}(k))\right) \\
& \cong 1 \quad R(G) \underset{R(T)}{\stackrel{L}{\otimes}}\left(\mathbf{G}_{0}([X / T]) \underset{R(T)}{\stackrel{L}{\otimes}} \mathbf{K}_{*}^{T}(\operatorname{Spec}(k))\right) \\
& \cong^{2}\left(R(G) \stackrel{L}{\otimes} \mathbf{G}_{0}([X / T])\right) \underset{R(T)}{\otimes}\left(R(T) \underset{\mathbb{Z}}{\otimes} \mathbf{K}_{*}(\operatorname{Spec}(k))\right)
\end{aligned}
$$

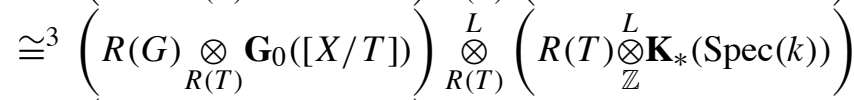

$$
\begin{aligned}
& \cong^{4}\left(R(G) \underset{R(T)}{\otimes} \mathbf{G}_{0}([X / T])\right) \underset{\mathbb{Z}}{\otimes} \mathbf{K}_{*}(\operatorname{Spec}(k)) \\
& \cong^{5}\left(R(G) \underset{R(T)}{\otimes} \mathbf{G}_{0}([X / T])\right) \underset{\mathbb{Z}}{\otimes} \mathbf{K}_{*}(\operatorname{Spec}(k)) \\
& \cong^{6} R(G) \underset{R(T)}{\otimes}\left(\mathbf{G}_{0}([X / T]) \underset{\mathbb{Z}}{\otimes \mathbf{K}_{*}}(\operatorname{Spec}(k))\right) \\
& \cong^{7} R(G) \underset{R(T)}{\otimes} \mathbf{G}_{*}([X / T]) \text {. }
\end{aligned}
$$

The isomorphism $\simeq 0$ follows from [41, Proposition 6.4] in general and also from Theorem 1.2 when $X$ is projective. The isomorphism $\simeq{ }^{1}$ follows because $\mathbf{G}_{0}([X / T])$ is projective $R(T)$-module. The isomorphism $\simeq^{2}$ follows from Lemma 3.11 because $R(T)$ is flat $\mathbb{Z}$-module. The isomorphism $\simeq^{3}$ follows again from the projectivity of $\mathbf{G}_{0}([X / T])$ as an $R(T)$-module. The isomorphisms $\simeq^{4}$ and $\simeq^{6}$ are the associativity of the ordinary and derived tensor products. The isomorphism $\simeq^{5}$ follows because $R(G)$ is a free $\mathbb{Z}$-module and $R(G) \underset{R(T)}{\otimes} \mathbf{G}_{0}([X / T])$ is a projective $R(G)$-module and hence is flat as a $\mathbb{Z}$-module. The isomorphism $\simeq^{7}$ follows again 
from [41, Proposition 6.4] in general and also from Theorem 1.2 when $X$ is projective. This proves (4.5). The projectivity of $\mathbf{G}_{0}([X / T])$ as $R(T)$-module when $X$ is a smooth and projective toric variety, is shown in [41, Proposition 6.9] (see also Lemma 6.1). The proof of Theorem 1.1 is now complete.

Remark 4.2. It was shown by Baggio [1] that there are examples of non-projective smooth toric varieties $X$ such that $\mathbf{G}_{0}([X / T])$ is a projective $R(T)$-module. This shows that there are smooth non-projective toric varieties for which the spectral sequence in Theorem 1.1 degenerates. In all these cases, one obtains a complete description of the K-theory of the toric stack $[X / G]$. We shall see in Section 5.2 that there are examples where the spectral sequence of Theorem 1.1 degenerates even if $\mathbf{G}_{0}([X / T])$ is not a projective $R(T)$-module.

The following generalization of [27, Theorem 4.3] follows immediately from Theorem 1.1, [34, Theorem 1.3] and [27, Proposition 4.1].

Corollary 4.3. Let $G$ be a connected and reductive group over $k$ with a split maximal torus $T$ such that $\pi_{1}(G)$ is torsion-free. Suppose that $G$ acts on scheme $X$. Let $\phi: H \rightarrow T$ be a morphism of diagonalizable groups. Then there is strongly convergent spectral sequence

$$
E_{2}^{s, t}=\operatorname{Tor}_{s}^{R(G)}\left(R(H), \mathbf{G}_{t}([X / G])\right) \Rightarrow \mathbf{G}_{s+t}([X / H]) .
$$

Moreover, the edge map $R(H) \underset{R(G)}{\otimes} \mathbf{G}_{0}([X / G]) \rightarrow \mathbf{G}_{0}([X / H])$ is an isomorphism.

\subsection{Grothendieck group of toric stacks}

In [4], Borisov and Horja had computed the Grothendieck $K$-theory ring $\mathbf{K}_{0}([X / G])$ when $[X / G]$ is a smooth toric Deligne-Mumford stack. Recall from Section 2 that the dense stacky torus of a Deligne-Mumford stack is of the form $T^{\prime} \times \mathfrak{B}_{\mu}$ where $T^{\prime}$ is a torus and $\mu$ is a finite abelian group. The following consequence of Theorem 1.1 generalizes the result of [4] to the case of all smooth toric stacks, not necessarily Deligne-Mumford. Even in this latter case, we obtain a simpler proof.

Theorem 4.4. Let $\mathfrak{X}=[X / G]$ be a smooth and reduced toric stack associated to the data $\underline{X}=(X, G \stackrel{\phi}{\rightarrow} T)$. Let $\Delta$ be the fan defining $X$ and let $d$ be the number of rays in $\Delta$. Let $I_{\Delta}^{G}$ denote the ideal of the Laurent polynomial algebra $\mathbb{Z}\left[t_{1}^{ \pm 1}, \cdots, t_{d}^{ \pm 1}\right]$ generated by the relations:

(1) $\left(t_{j_{1}}-1\right) \cdots\left(t_{j_{l}}-1\right), 1 \leq j_{p} \leq d$ such that the rays $\rho_{j_{1}}, \cdots, \rho_{j_{l}}$ do not span a cone of $\Delta$;

(2) $\left(\prod_{j=1}^{d}\left(t_{j}\right)^{<-\chi, v_{j}>}\right)-1, \chi \in(T / G)^{\vee}$. 
Then there is a ring isomorphism

$$
\phi: \frac{\mathbb{Z}\left[t_{1}^{ \pm 1}, \cdots, t_{d}^{ \pm 1}\right]}{I_{\Delta}^{G}} \stackrel{\simeq}{\rightarrow} \mathbf{K}_{0}(\mathfrak{X}) .
$$

Proof. It follows from Theorem 1.1 that the map $\mathbf{K}_{0}([X / T]) \underset{R(T)}{\otimes R}(G) \stackrel{\sim}{\rightarrow} \mathbf{K}_{0}([X / G])$ is a ring isomorphism. Since $G$ is a diagonalizable subgroup of $T$ ([X/G] is reduced), the ring $R(G)$ is a quotient of $R(T)$ by the ideal $J_{\Delta}^{G}=\left(\chi-1, \chi \in(T / G)^{\vee}\right)$ (cf. Lemma 7.8). This implies that

$$
\mathbf{K}_{0}([X / G]) \simeq \frac{\mathbf{K}_{0}([X / T])}{J_{\Delta}^{G} \mathbf{K}_{0}([X / T])} .
$$

If we let $\Delta(1)=\left\{\rho_{1}, \cdots, \rho_{d}\right\}$, then for each $1 \leq j \leq d$, there is a unique $T$ equivariant line bundle $L_{j}$ on $X$ which has a $T$-equivariant section $s_{j}: X \rightarrow L_{j}$ and whose zero locus is the orbit closure $V_{j}=\overline{O_{\rho_{j}}}$. Then every character $\chi \in T^{\vee}$ acts on $\mathbf{K}_{0}([X / T])$ by multiplication with the element $\left(\prod_{j=1}^{d}\left(\left[L_{j}\right]\right)^{<\chi, v_{j}>}\right)(c f$. [32, Proposition 4.3]). We conclude that there is a ring isomorphism

$$
\frac{\mathbf{K}_{0}([X / T])}{\left(\prod_{j=1}^{d}\left(\left[L_{j}^{\vee}\right]\right)^{<-\chi, v_{j}>}-1, \chi \in(T / G)^{\vee}\right)} \stackrel{\cong}{\rightarrow} \mathbf{K}_{0}([X / G]) .
$$

If $I_{\Delta}^{T}$ denotes the ideal of $\mathbb{Z}\left[t_{1}^{ \pm 1}, \cdots, t_{d}^{ \pm 1}\right]$ generated by the relations (1) above, then it follows from [41, Theorem 6.4] that there is a ring isomorphism

$$
\frac{\mathbb{Z}\left[t_{1}^{ \pm 1}, \cdots, t_{d}^{ \pm 1}\right]}{I_{\Delta}^{T}} \stackrel{\simeq}{\rightarrow} \mathbf{K}_{0}([X / T]) .
$$

Setting $\phi\left(t_{j}\right)=\left[L_{j}^{\vee}\right]$, we obtain the isomorphism (4.7) by combining (4.9) and (4.10).

Remark 4.5. If $[X / G]$ is not a reduced stack and there is an exact sequence

$$
0 \rightarrow H \rightarrow G \rightarrow F \rightarrow 0
$$

where $F=\operatorname{Im}(\phi)$, then the stack $[X / G]$ is isomorphic to $[X / F] \times \mathfrak{B}_{H}$. In this case, one obtains an isomorphism $\mathbf{K}_{*}([X / G]) \simeq \mathbf{K}_{*}([X / F]) \underset{R(F)}{\otimes} R(G)(c f$. Lemma 3.11). In particular, if $H$ is a torus, one obtains

$$
\mathbf{K}_{*}([X / G]) \simeq \mathbf{K}_{*}([X / F]) \underset{\mathbb{Z}}{\otimes} R(H) .
$$

Thus, we see that the calculation of the K-theory of a (generically stacky) toric stack can be easily reduced to the case of reduced stacks. 


\section{A Künneth formula and its consequences}

Our goal in this section is to prove Theorem 1.2 and give applications. We shall deduce this theorem from a Künneth spectral sequence for the equivariant K-theory for the action of diagonalizable groups. A spectral sequence of this kind in the non-equivariant setting was constructed by the first author in [20, Theorem 4.1].

\subsection{Künneth formula}

Suppose that $X$ and $X^{\prime}$ are schemes acted upon by a linear algebraic group $G$. In this case, the flatness of $X$ and $X^{\prime}$ over $k$ implies that the spectra $\mathbf{G}([X / G])$ and $\mathbf{G}\left(\left[X^{\prime} / G\right]\right)$ are module spectra over the ring spectrum $\mathbf{K}([\operatorname{Spec}(k) / G])$. This flatness also ensures that the external tensor product of coherent $\mathcal{O}$-modules induces a pairing $\mathbf{G}([X / G]) \wedge \mathbf{G}\left(\left[X^{\prime} / G\right]\right) \rightarrow \mathbf{G}\left(\left[\left(X \times X^{\prime}\right) / G\right]\right)$, where the action of $G$ on $X \times X^{\prime}$ is the diagonal action. This pairing is compatible with the structure of the above spectra as module spectra over the ring spectrum $\mathbf{K}([\operatorname{Spec}(k) / G])$ so that one obtains the induced pairing:

$$
p_{1}^{*} \wedge p_{2}^{*}: \mathbf{G}([X / G]) \underset{\mathbf{K}([\operatorname{Spec}(k) / G])}{\stackrel{L}{\wedge}} \mathbf{G}\left(\left[X^{\prime} / G\right]\right) \rightarrow \mathbf{G}\left(\left[\left(X \times X^{\prime}\right) / G\right]\right) .
$$

This is a map of ring spectra if $X$ and $X^{\prime}$ are smooth.

Proposition 5.1. Let $T$ be a split torus and let $X, X^{\prime}$ be in $\mathcal{V}_{T}$ such that $X$ is $T$ linear. Let $\phi: G \rightarrow T$ be a morphism of diagonalizable groups such that $G$ acts on $X$ and $X^{\prime}$ via $\phi$. Then the natural map of spectra

$$
\mathbf{G}([X / G]) \underset{\mathbf{K}([\operatorname{Spec}(k) / G])}{\stackrel{L}{\wedge}} \mathbf{G}\left(\left[X^{\prime} / G\right]\right) \rightarrow \mathbf{G}\left(\left[\left(X \times X^{\prime}\right) / G\right]\right)
$$

is a weak equivalence.

In particular, there exists a first quadrant spectral sequence

$$
E_{s, t}^{2}=\operatorname{Tor}_{s, t}^{\mathbf{K}_{*}^{G}(k)}\left(\mathbf{G}_{*}([X / G]), \mathbf{G}_{*}\left(\left[X^{\prime} / G\right]\right)\right) \Rightarrow \mathbf{G}_{s+t}\left(\left[\left(X \times X^{\prime}\right) / G\right]\right) .
$$

Proof. We assume that $X$ is $T$-equivariantly $n$-linear for some $n \geq 0$. The proposition is proved by an ascending induction on $n$, along the same lines as the proof of Proposition 3.10. We sketch the argument.

If $n=0$, then $X \simeq \mathbb{A}^{n}$ and hence by the homotopy invariance, we can assume that $X=\operatorname{Spec}(k)$, and the result is immediate in this case. We now assume that $n>0$. By the definition of $T$-linearity, there are two cases to consider:

(1) There exists a $T$-invariant closed subscheme $Y$ of $X$ with complement $U$ such that $Y$ and $U$ are $T$-equivariantly $(n-1)$-linear.

(2) There exists a $T$-scheme $Z$ which contains $X$ as a $T$-invariant open subscheme such that $Z$ and $Y=Z \backslash X$ are $T$-equivariantly $(n-1)$-linear. 
In the first case, the localization fiber sequence in equivariant G-theory gives us a commutative diagram of fiber sequences in the homotopy category of spectra:

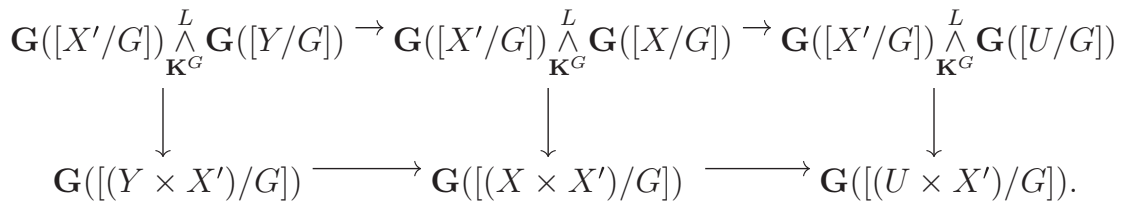

The left and the right vertical maps are weak equivalences by the induction on $n$. We conclude that the middle vertical map is a weak equivalence. The second case is proved in the same way where we now use induction on $Y$ and $Z$ (see the proof of Proposition 3.10). The existence of the spectral sequence now follows from (3.5).

Remark 5.2. As an application of Proposition 5.1, one can obtain another proof of the special case of the spectral sequence (1.1) when $G$ is a closed subgroup of $T$. This is done by taking $G=T, X^{\prime}=T / G$ in (5.2) and using the Morita weak equivalences $\mathbf{G}\left(\left[X^{\prime} / T\right]\right) \simeq \mathbf{G}([\operatorname{Spec}(k) / G])$ and $\mathbf{G}\left(\left[\left(X \times X^{\prime}\right) / T\right]\right) \simeq \mathbf{G}([X / G])$. Notice that $X^{\prime}=T / G$ is $T$-linear by Proposition 3.6.

Corollary 5.3 (Künneth decomposition). Let $T$ be a split torus over $k$ and let $X$ be a $T$-linear scheme. Then the class of the diagonal $[\Delta] \in \mathbf{G}_{0}([(X \times X) / G])$ admits a strong Künneth decomposition, i.e., may be written as $\Sigma_{i=1}^{n} p_{1}^{*}\left(\alpha_{i}\right) \otimes p_{2}^{*}\left(\beta_{i}\right)$, where $\alpha_{i}, \beta_{i} \in \mathbf{G}_{0}([X / G])$.

Proof. The spectral sequence of Proposition 5.1 shows in general that

$$
\mathbf{G}_{0}\left(\left[\left(X \times X^{\prime}\right) / G\right]\right) \simeq \mathbf{G}_{0}([X / G]) \underset{R(G)}{\otimes} \mathbf{G}_{0}\left(\left[X^{\prime} / G\right]\right) .
$$

The Künneth decomposition now follows by taking $X=X^{\prime}$.

Proof of Theorem 1.2. Let $X$ be a smooth and projective $T$-linear scheme. Since the group $G$ is diagonalizable, we apply Lemma 3.11 to obtain the isomorphism:

$$
R(G) \underset{\mathbb{Z}}{\otimes \mathbf{K}_{*}}(k) \stackrel{\simeq}{\rightarrow} \mathbf{K}_{*}^{G}(k)
$$

and this provides the first isomorphism of (1.3). Since $X$ is smooth, we can identify $G_{*}([X / G])$ with $\mathbf{K}_{*}([X / G])$.

Let $[x] \in \mathbf{K}_{*}([X / G])$. Then $[x]=p_{1 *}\left(\Delta \circ p_{2}^{*}([x])\right)$. Now we use the Künneth decomposition for $\Delta$ obtained in Corollary 5.3 and the projection formula (since $X$ is projective) to identify the last term with $\sum_{i=1}^{n} \alpha_{i} \circ p_{1 *} p_{2}^{*}\left(\beta_{i} \circ[x]\right)$. The Cartesian square

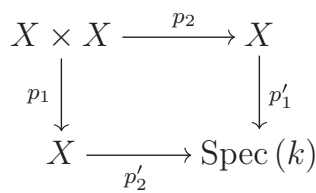


and the flat base-change for the equivariant G-theory show that $p_{1 *}\left(p_{2}^{*}\left(\beta_{i} \circ[x]\right)\right)$ identifies with $p_{2}^{\prime *} p_{1 *}^{\prime}\left(\beta_{i} \circ[x]\right)$ so that

$$
[x]=\sum_{i=1}^{n} \alpha_{i} \circ p_{2}^{\prime *}\left(p_{1 *}^{\prime}\left(\beta_{i} \circ[x]\right)\right) .
$$

The class $p_{1 *}^{\prime}\left(\beta_{i} \circ[x]\right) \in \mathbf{G}([\operatorname{Spec}(k) / G])$. It follows that the classes $\left\{\alpha_{i}\right\}$ generate $\pi_{*}(G[X / G])$ as a module over $\mathbf{K}_{*}^{G}(k)$. This shows that the map in question is surjective.

Next we prove the injectivity of the map $\rho$. The key is the following diagram:

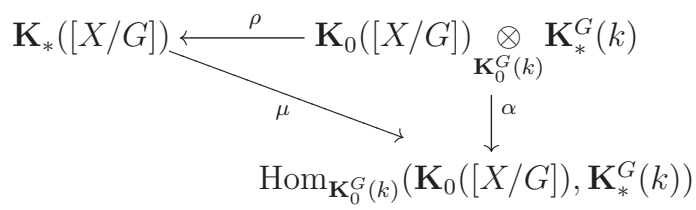

where $\alpha(x \otimes y)$ (respectively $\left.\mu(x), x \in \mathbf{K}_{*}([X / G])\right)$ is defined by $\alpha(x \otimes y)=$ the map $x^{\prime} \mapsto f_{*}\left(x^{\prime} \circ x\right) \circ y$ (respectively, the map $\left.x^{\prime} \mapsto f_{*}\left(x^{\prime} \circ x\right)\right)$. Here, $f$ denotes the projection map $X \rightarrow \operatorname{Spec}(k)$ and $x^{\prime} \circ x$ denotes the product in the ring $\mathbf{K}_{*}([X / G])$. The commutativity of the above diagram is an immediate consequence of the projection formula: observe that $\rho(x \otimes y)=x \circ f^{*}(y)$. Therefore, to show that $\rho$ is injective, it suffices to show that the map $\alpha$ is injective. For this, we define a map $\beta$ to be a splitting for $\alpha$ as follows.

If $\phi \in \operatorname{Hom}_{\mathbf{K}_{0}^{G}(k)}\left(\mathbf{K}_{0}([X / G]), \mathbf{K}_{*}^{G}(k)\right)$, we let $\beta(\phi)=\sum_{i=1}^{n} \alpha_{i} \otimes\left(\phi\left(\beta_{i}\right)\right)$. Observe that

$$
\begin{aligned}
\beta(\alpha(x \otimes y)) & =\beta\left(\text { the } \operatorname{map} \quad x^{\prime} \rightarrow f_{*}\left(x^{\prime} \circ x\right) \circ y\right) \\
& =\left(\sum_{i=1}^{n} \alpha_{i} \otimes f_{*}\left(\beta_{i} \cdot x\right)\right) \circ y .
\end{aligned}
$$

We next observe that $f_{*}\left(\beta_{i} \cdot x\right) \in \mathbf{K}_{0}^{G}(k)$, so that we may write the last term as $\left(\sum_{i=1}^{n} \alpha_{i} \cdot f^{*} f_{*}\left(\beta_{i} \cdot x\right)\right) \circ y$. By (5.5), the last term $=x \circ y$. This proves that $\alpha$ is injective and hence that so is $\rho$. This completes the proof.

The following result generalizes (1.2) to a bigger class of schemes.

Corollary 5.4. Let $T$ be a split torus over $k$ and let $X$ be a smooth and projective $T$-linear scheme. Let $\phi: G \rightarrow T$ be a morphism of diagonalizable groups such that $G$ acts on $X$ via $\phi$. Then the map

$$
R(G) \underset{R(T)}{\otimes} \mathbf{K}_{*}([X / T]) \rightarrow \mathbf{K}_{*}([X / G])
$$

is an isomorphism. In particular, $\mathbf{K}_{0}([X / G])$ is a free $R(G)$-module (and hence a free $\mathbb{Z}$-module) if $X$ is $T$-cellular. 
Proof. To prove the first part of the corollary, we trace through the sequence of isomorphisms:

$$
\begin{aligned}
R(G) \underset{R(T)}{\otimes} \mathbf{K}_{*}([X / T]) & \cong R(G) \underset{R(T)}{\otimes}\left(\mathbf{K}_{0}([X / T]) \underset{R(T)}{\otimes} \mathbf{K}_{*}^{T}(\operatorname{Spec}(k))\right) \\
& \cong\left(R(G) \underset{R(T)}{\otimes} \mathbf{K}_{0}([X / T])\right) \underset{R(T)}{\otimes}\left(R(T) \underset{\mathbb{Z}}{\otimes} \mathbf{K}_{*}(\operatorname{Spec}(k))\right) \\
& \cong \mathbf{K}_{0}([X / G]) \underset{\mathbb{Z}}{\otimes} \mathbf{K}_{*}(\operatorname{Spec}(k)) \\
& \cong \mathbf{K}_{*}([X / G]) .
\end{aligned}
$$

The first and the last isomorphisms in this sequence follow from Theorem 1.2 and the isomorphism $\simeq^{\dagger}$ follows from Theorem 1.1. This proves the first part of the corollary. If $X$ is $T$-cellular, the freeness of $\mathbf{K}_{0}([X / G])$ as an $R(G)$-module follows from Lemma 6.1.

Remark 5.5. In the special case when $[X / G]$ is a smooth toric Deligne-Mumford stack (with $X$ projective), the freeness of $\mathbf{K}_{0}([X / G])$ as a $\mathbb{Z}$-module was earlier shown in [17, Theorem 2.2] and independently in [13] using symplectic methods. It is known ( $c f$. [17, Example 4.1]) that the freeness property may fail if $X$ is not projective.

\subsection{K-theory of weighted projective spaces}

In the past, there have been many attempts to study the K-theory and Chow rings of weighted projective spaces. However, there are only a few explicit computations in this regard. We end this section with an explicit description of the integral higher K-theory of stacky weighted projective spaces. These are examples of toric stacks, where the spectral sequence (1.1) degenerates even though $\mathbf{K}_{0}([X / T])$ is not a projective $R(T)$-module. We also describe the rational higher G-theory of weighted projective schemes as another application of Theorem 1.2.

\subsubsection{Weighted projective spaces}

Let $\underline{q}=\left\{q_{0}, \cdots, q_{n}\right\}$ be an ordered set of positive integers and let $d=\operatorname{gcd}\left(q_{0}, \cdots, q_{n}\right)$. This ordered set of positive integers gives rise to a morphism of tori $\phi: \mathbb{G}_{m} \rightarrow$ $\left(\mathbb{G}_{m}\right)^{n+1}$ given by $\phi(\lambda)=\left(\lambda^{q_{0}}, \cdots, \lambda^{q_{n}}\right)$.

The (stacky) weighted projective space $\mathbb{P}\left(q_{0}, \cdots, q_{n}\right)$ is the stack

$$
\left[\left(\mathbb{A}_{k}^{n+1} \backslash\{0\}\right) / \mathbb{G}_{m}\right],
$$

where $\mathbb{G}_{m}$ acts on $\mathbb{A}_{k}^{n+1}$ by $\lambda \cdot\left(a_{0}, \cdots, a_{n}\right)=\left(\lambda^{q_{0}} a_{0}, \cdots, \lambda^{q_{n}} a_{n}\right)$. Notice that $\mathbb{A}^{n+1} \backslash\{0\}$ is a toric variety with dense torus $T=\left(\mathbb{G}_{m}\right)^{n+1}$ acting by the coordinatewise multiplication. We see that $\mathbb{P}(\underline{q})$ is the toric stack associated to the data $\left(\left(\mathbb{A}_{k}^{n+1} \backslash\{0\}\right), \mathbb{G}_{m} \stackrel{\phi}{\rightarrow} T\right)$. It is known that $\mathbb{P}(\underline{q})$ is a Deligne-Mumford toric stack and is reduced (an orbifold) if and only if $d=1$. 


\subsubsection{K-theory of $\mathbb{P}(q)$}

To describe the higher K-theory of $\mathbb{P}(\underline{q})$, we consider $\mathbb{A}^{n+1}$ as the toric variety with dense torus $T=\left(\mathbb{G}_{m}\right)^{n+1}$ acting by the coordinate-wise multiplication. Let $V$ be the $(n+1)$-dimensional representation of $T$ which represents $\mathbb{A}^{n+1}$ as the toric variety. Let $\iota: \operatorname{Spec}(k) \rightarrow \mathbb{A}^{n+1}$ and $j: U \rightarrow \mathbb{A}^{n+1}$ be the $T$-invariant closed and open inclusions, where we set $U=\mathbb{A}^{n+1} \backslash\{0\}$. Observe that $V$ is the $T$-equivariant normal bundle of $\operatorname{Spec}(k)$ sitting inside $\mathbb{A}^{n+1}$ as the origin.

We have the localization exact sequence:

$$
\cdots \rightarrow \mathbf{K}_{i}\left(\left[\operatorname{Spec}(k) / \mathbb{G}_{m}\right]\right) \stackrel{\iota_{*}}{\rightarrow} \mathbf{K}_{i}\left(\left[\mathbb{A}^{n+1} / \mathbb{G}_{m}\right]\right) \stackrel{j^{*}}{\rightarrow} \mathbf{K}_{i}\left(\left[U / \mathbb{G}_{m}\right]\right) \rightarrow \cdots .
$$

Our first claim is that this sequence splits into short exact sequences

$$
0 \rightarrow \mathbf{K}_{i}\left(\left[\operatorname{Spec}(k) / \mathbb{G}_{m}\right]\right) \stackrel{\iota_{*}}{\rightarrow} \mathbf{K}_{i}\left(\left[\mathbb{A}^{n+1} / \mathbb{G}_{m}\right]\right) \stackrel{j^{*}}{\rightarrow} \mathbf{K}_{i}\left(\left[U / \mathbb{G}_{m}\right]\right) \rightarrow 0
$$

for each $i \geq 0$.

Using [41, Proposition 4.3], it suffices to show that

$$
\lambda_{-1}(V)=\sum_{i=0}^{n}(-1)^{i}\left[\wedge^{i}(V)\right]
$$

is not a zero-divisor in the ring $\mathbf{K}_{*}\left(\left[\operatorname{Spec}(k) / \mathbb{G}_{m}\right]\right)$. However, we can write $V=\underset{i=0}{\oplus}$ $V_{i}$, where $\mathbb{G}_{m}$ acts on $V_{i} \simeq k$ by $\lambda \cdot v=\lambda^{q_{i}} v$. Since each $q_{i}$ is positive, we see that no irreducible factor of $V$ is trivial. It follows from [41, Lemma 4.2] that $\lambda_{-1}(V)$ is not a zero-divisor in the ring $\mathbf{K}_{*}\left(\left[\operatorname{Spec}(k) / \mathbb{G}_{m}\right]\right)$, and hence (5.8) is exact. We have thus proven our claim.

We can now use (5.8) to compute $\mathbf{K}_{*}\left(\left[U / \mathbb{G}_{m}\right]\right)$. We first observe that the $\operatorname{map} \mathbf{K}_{*}\left(\left[\operatorname{Spec}(k) / \mathbb{G}_{m}\right]\right) \rightarrow \mathbf{K}_{*}\left(\left[\mathbb{A}^{n+1} / \mathbb{G}_{m}\right]\right)$ induced by the structure map is an isomorphism by the homotopy invariance. So we can identify the middle term of (5.8) with $\mathbf{K}_{i}\left(\left[\operatorname{Spec}(k) / \mathbb{G}_{m}\right]\right)$. Furthermore, it follows from the Self-intersection formula [41, Theorem 2.1] that the map $\iota_{*}$ is multiplication by $\lambda_{-1}(V)$ under this identification.

Since $V=\bigoplus_{i=0}^{n} V_{i}$, we get $\lambda_{-1}(V)=\prod_{i=0}^{n} \lambda_{-1}\left(V_{i}\right)$. Furthermore, since the class of $V_{i}$ in $R\left(\mathbb{G}_{m}\right)=\mathbb{Z}\left[t^{ \pm 1}\right]$ is $t^{q_{i}}$, we see that $\lambda_{-1}\left(V_{i}\right)=1-t^{q_{i}}$. We conclude that $\lambda_{-1}(V)=\prod_{i=0}^{n}\left(1-t^{q_{i}}\right)$. We have thus proven:

Theorem 5.6. There is a ring isomorphism

$$
\frac{\mathbf{K}_{*}(k)\left[t^{ \pm 1}\right]}{\prod_{i=0}^{n}\left(1-t^{q_{i}}\right)} \stackrel{\cong}{\longrightarrow} \mathbf{K}_{*}(\mathbb{P}(\underline{q})) .
$$

Remark 5.7. In the above calculations, we can replace $\mathbb{G}_{m}$ by the dense torus $T$ to get a similar formula. In this case, the exact sequence (5.8) shows that $\mathbf{K}_{0}\left(\left[\left(\mathbb{A}^{n+1} \backslash\right.\right.\right.$ $\{0\}) / T]$ ) is a quotient of $R(T)$ and hence is not a projective $R(T)$-module. 


\subsubsection{G-theory of weighted projective scheme}

The weighted projective scheme is the scheme theoretic quotient of $\mathbb{A}^{n+1} \backslash\{0\}$ by the above action of $\mathbb{G}_{m}$. This is the coarse moduli scheme of $\mathbb{P}(q)$. We shall denote this scheme by $\widetilde{\mathbb{P}(q)}$. It is known that this is a normal (but singular in general) projective scheme. There was no computation available for the higher G-theory or K-theory of this schematic weighted projective space. As an application of Theorem 1.2, we now give a simple description of the rational higher G-theory of $\widetilde{P}(q)$. We still do not know how to compute its K-theory. (See [25] for very partial results in this direction.)

In order to describe the higher G-theory of $\widetilde{\mathbb{P}(q)}$, we shall use the following presentation of this scheme which allows us to use our main results. We assume that the characteristic of $k$ does not divide any $q_{i}$.

The torus $T=\mathbb{G}_{m}^{n}$ acts on $\mathbb{P}_{k}^{n}$ as the dense open torus by $\left(\lambda_{1}, \cdots, \lambda_{n}\right) \star$ $\left[z_{0}, \cdots, z_{n}\right]=\left[z_{0}, \lambda_{1} z_{1}, \cdots, \lambda_{n} z_{n}\right]$. Let $G=\mu_{q_{0}} \times \cdots \times \mu_{q_{n}}$ be the product of finite cyclic groups. Then $G$ acts on $\mathbb{P}_{k}^{n}$ by $\left(a_{0}, \cdots, a_{n}\right) \bullet\left[z_{0}, \cdots, z_{n}\right]=$ $\left[a_{0} z_{0}, \cdots, a_{n} z_{n}\right]$. It is then easy to see that $\widetilde{\mathbb{P}(q)}$ is isomorphic to the scheme $\mathbb{P}_{k}^{n} / G$.

Define $\phi: G \rightarrow T$ by $\phi\left(a_{0}, \cdots, a_{n}\right)=\left(a_{1} / a_{0}, \cdots, a_{n} / a_{0}\right)$. Then one checks that

$$
\begin{aligned}
H:=\operatorname{Ker}(\phi) & =\left\{\left(a_{0}, \cdots, a_{n}\right) \in G \mid a_{0}=\cdots=a_{n}\right\} \\
& =\left\{\lambda \in \mathbb{G}_{m} \mid \lambda^{q_{0}}=1=\cdots=\lambda^{q_{n}}\right\} \\
& =\left\{\lambda \in \mathbb{G}_{m} \mid \lambda^{d}=1\right\} \\
& \simeq \mu_{d} .
\end{aligned}
$$

Moreover, it is easy to see that

$$
\begin{aligned}
\left(a_{0}, \cdots, a_{n}\right) \bullet\left[z_{0}, \cdots, z_{n}\right] & =\left[a_{0} z_{0}, \cdots, a_{n} z_{n}\right] \\
& =\left[a_{0}^{-1}\left(a_{0} z_{0}\right), \cdots, a_{0}^{-1}\left(a_{n} z_{n}\right)\right] \\
& =\left[z_{0},\left(a_{1} / a_{0}\right) z_{1}, \cdots,\left(a_{n} / a_{0}\right) z_{n}\right] \\
& =\phi\left(a_{0}, \cdots, a_{n}\right) \star\left[z_{0}, \cdots, z_{n}\right] .
\end{aligned}
$$

In particular, $G$ acts on $\mathbb{P}_{k}^{n}$ through $\phi$. We conclude that $\mathfrak{X}=\left[\mathbb{P}_{k}^{n} / G\right]$ is a smooth toric Deligne-Mumford stack associated to the data $\left(\mathbb{P}_{k}^{n}, G \stackrel{\phi}{\rightarrow} T\right)$ and there is an isomorphism $\mathfrak{X} \simeq\left[\mathbb{P}_{k}^{n} / F\right] \times \mathfrak{B}_{\mu_{d}}$, where $F=\operatorname{Im}(\phi)$.

Theorem 5.8. There is a ring isomorphism

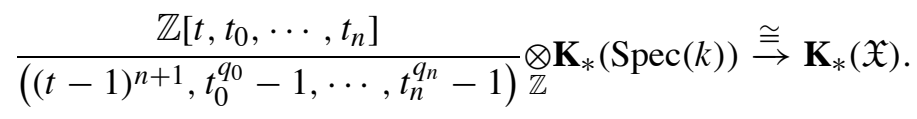

Proof. It follows from Corollary 5.4 and Theorem 1.2 that there is a ring isomorphism

$$
\left.R(G) \underset{R(T)}{\otimes} \mathbf{K}_{0}\left(\left[\mathbb{P}_{k}^{n} / T\right]\right) \underset{\mathbb{Z}}{\otimes \mathbf{K}_{*}}(\operatorname{Spec}(k)) \stackrel{\cong}{\rightarrow} \mathbf{K}_{*}(\mathfrak{X})\right) .
$$


On the other hand, the projective bundle formula implies that the left side of this isomorphism is same as $R(G) \underset{R(T)}{\otimes} \frac{R(T)[t]}{\left((t-1)^{n+1}\right)} \underset{\mathbb{Z}}{\otimes \mathbf{K}_{*}}(\operatorname{Spec}(k))$, which in turn is isomorphic to $\frac{R(G)[t]}{\left((t-1)^{n+1}\right)} \underset{\mathbb{Z}}{\otimes \mathbf{K}_{*}}(\operatorname{Spec}(k))$. The theorem now follows from the isomorphism $R(G) \simeq \frac{\mathbb{Z}\left[t_{0}, \cdots, t_{n}\right]}{\left(t_{0}^{q_{0}}-1, \cdots, t_{n}^{q_{n}}-1\right)}$.

Corollary 5.9. There is an isomorphism

$$
\frac{\mathbf{G}_{*}(k)[t]}{\left((t-1)^{n+1}\right)} \stackrel{\simeq}{\rightarrow} \mathbf{G}_{*}(\widetilde{\mathbb{P}(\underline{q})})
$$

with rational coefficients.

Proof. All the groups in this proof will be considered with rational coefficients. Let $\pi: \mathbb{P}_{k}^{n+1} \rightarrow \widetilde{\mathbb{P}(\underline{q})}$ be the quotient map. The assignment $\mathcal{F} \mapsto\left(\pi_{*}(\mathcal{F})\right)^{G}$ defines a covariant functor from the category of $G$-equivariant coherent sheaves on $\mathbb{P}_{k}^{n+1}$ to the category of ordinary coherent sheaves on $\widetilde{\mathbb{P}(q)}$. Since the characteristic of $k$ does not divide the order of $G$, this functor is exact and gives a push-forward map $\pi_{*}: \mathbf{G}_{*}^{G}\left(\mathbb{P}_{k}^{n+1}\right) \rightarrow \mathbf{G}_{*}(\widetilde{\mathbb{P}(\underline{q})})$.

Let $\mathrm{CH}_{*}^{G}\left(\mathbb{P}_{k}^{n+1}\right)$ denote the equivariant higher Chow groups of $\mathbb{P}_{k}^{n+1}$ ( [9]). By [9, Theorem 3], there is a push-forward map $\bar{\pi}_{*}: \mathrm{CH}_{*}^{G}\left(\mathbb{P}_{k}^{n+1}\right) \rightarrow \mathrm{CH}_{*}(\widetilde{\mathbb{P}(q)})$ which is an isomorphism. It follows from [22, Theorem 9.8, Lemma 9.1] that there is a commutative diagram

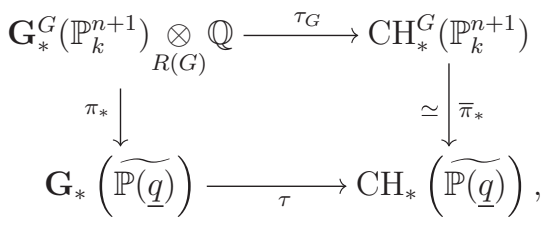

where the horizontal arrows are the Riemann-Roch maps which are isomorphisms ( $[22$, Theorem 8.6]). It follows that the left vertical arrow is an isomorphism. The corollary now follows by combining this isomorphism with Theorem 5.8.

\section{Toric stack bundles and the stacky Leray-Hirsch theorem}

Toric bundle schemes and their cohomology were first studied by Sankaran and Uma in [32]. They computed the Grothendieck group of a toric bundle over a smooth base scheme. A description of the Grothendieck group of toric DeligneMumford stack bundles was given by Jiang and Tseng in [19].

In this section, we give a general definition of toric stack bundles over a base scheme in such a way that every fiber of this bundle is a (generically stacky) toric 
stack in the sense of [14]. We prove a stacky version of the Leray-Hirsch theorem for the algebraic K-theory of stack bundles. This Leray-Hirsch theorem will be used in the next section to describe the higher K-theory of toric stack bundles.

\subsection{Toric stack bundles}

Let $T$ be a split torus of rank $n$ and let $X$ be a scheme with a $T$-action. Let $G$ be a diagonalizable group over $k$ and let $\phi: G \rightarrow T$ be a morphism of algebraic groups over $k$.

Let $p: E \rightarrow B$ be a principal $T$-bundle over a scheme $B$. Let $G$ act on $E \times X$ by $g(e, x)=(e, g x):=(e, \phi(g) x)$ and let $T$ act on $E \times X$ via the diagonal action. It is easy to see that these two actions commute and the projection map $E \times X \rightarrow E$ is equivariant with respect to these actions.

The commutativity of the actions ensures that the $G$-action descends to the quotients $E(X):=E \stackrel{T}{\times} X$ and $E / T=B$ such that the induced map of quotients $\bar{p}: E(X) \rightarrow B$ is $G$-equivariant. Since $E$ has trivial $G$-action, so does $B$ and we see that $G$ acts on $E(X)$ fiber-wise and the map $\bar{p}$ canonically factors through the stack quotient $\pi:[E(X) / G] \rightarrow B$. Notice that $E$ is a Zariski locally trivial $T$-bundle and so are $E(X) \rightarrow B$ and $[E(X) / G] \rightarrow B$. Setting $\mathfrak{X}=[E(X) / G]$, we conclude that the map $\pi: \mathfrak{X} \rightarrow B$ is a Zariski locally trivial fibration each of whose fiber is the stack $[X / G]$. The morphism $\pi$ will be called a stack bundle over $B$.

If $X$ is a toric variety with dense torus $T$, then $\pi: \mathfrak{X} \rightarrow B$ will be called a toric stack bundle over $B$. In this case, each fiber of $\pi$ is the toric stack $[X / G]$ in the sense of [14]. If $[X / G]$ is a Deligne-Mumford stack, this construction recovers the notion of toric stack bundles used in [19].

\subsection{Leray-Hirsch theorem for stack bundles}

First we prove the following lemma.

Lemma 6.1. Let $X$ be a $T$-equivariantly cellular scheme with the $T$-equivariant cellular decomposition

$$
\emptyset=X_{n+1} \subsetneq X_{n} \subsetneq \cdots \subsetneq X_{1} \subsetneq X_{0}=X
$$

and let $U_{i}=X \backslash X_{i}$ for $0 \leq i \leq n+1$. Let $G$ be a diagonalizable group provided with a morphism of algebraic groups $\phi: G \rightarrow T$. Then for any $0 \leq i \leq n$, the sequence

$$
0 \rightarrow \mathbf{G}_{*}^{G}\left(U_{i+1} \backslash U_{i}\right) \rightarrow \mathbf{G}_{*}^{G}\left(U_{i+1}\right) \rightarrow \mathbf{G}_{*}^{G}\left(U_{i}\right) \rightarrow 0
$$

is exact. In particular, $\mathbf{G}_{0}^{G}(X)$ is a free $R(G)$-module of rank equal to the number of $T$-invariant affine cells in $X$ with basis given by the closures of the affine cells.

Proof. To prove the exactness part of the proposition, we first make the following claim. Suppose $X$ is a $G$-scheme and $j: U \hookrightarrow X$ is a $G$-invariant open inclusion 
with complement $Y$. Suppose that $U$ is isomorphic to a representation of $G$. Then the localization sequence

$$
0 \rightarrow \mathbf{G}_{*}^{G}(Y) \rightarrow \mathbf{G}_{*}^{G}(X) \stackrel{j^{*}}{\rightarrow} \mathbf{G}_{*}^{G}(U) \rightarrow 0
$$

is (split) short exact.

To prove the claim, let $\alpha: X \rightarrow \operatorname{Spec}(k)$ and $\beta: U \rightarrow \operatorname{Spec}(k)$ be the structure maps (which are $G$-equivariant) so that $\beta=\alpha \circ j$. The homotopy invariance of equivariant $\mathrm{K}$-theory shows that $\beta^{*}$ is an isomorphism. Let $\gamma=\alpha^{*} \circ\left(\beta^{*}\right)^{-1}$. Then one checks that $\gamma$ is a section of $j^{*}$ and hence the localization sequence splits into short exact sequences. This proves the claim.

We shall prove (6.2) by induction on the number of $T$-invariant affine cells in $X$. For $i=0,(6.2)$ is immediate. So we assume $i \geq 1$ and consider the commutative diagram:

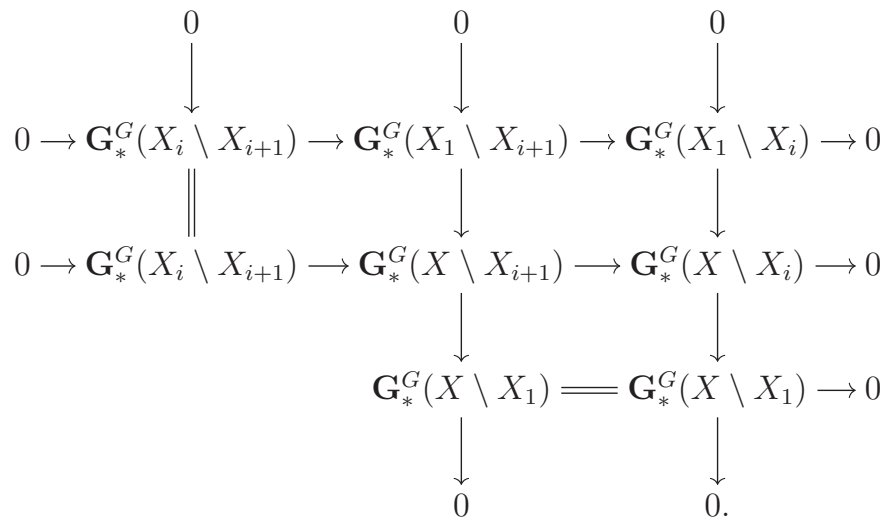

The top row is exact by induction on the number of affine cells since $X_{1}$ is $T$ equivariantly cellular with fewer number of cells. The two columns are exact by the above claim. It follows that the middle row is exact, which proves (6.2).

To prove the last (freeness) assertion, we apply (6.3) to the inclusion $X_{1} \subset X$ and see that $\mathbf{G}_{0}^{G}(X) \simeq \mathbf{G}_{0}^{G}\left(X_{1}\right) \oplus R(G)$. An induction on the number of affine $G$-cells now finishes the proof.

Proposition 6.2. Let $X$ be a $T$-equivariantly cellular scheme and let $B$ be any scheme with trivial $T$-action. Then the external product map

$$
\mathbf{G}_{0}^{G}(X) \otimes_{\mathbb{Z}} \mathbf{G}_{*}(B) \rightarrow \mathbf{G}_{*}^{G}(B \times X)
$$

is an isomorphism. In particular, the natural map $\mathbf{G}_{0}^{G}(X) \otimes_{\mathbb{Z}} \mathbf{K}_{*}(\operatorname{Spec}(k)) \rightarrow$ $\mathbf{G}_{*}^{G}(X)$ is an isomorphism.

Proof. Since the map

$$
R(G) \otimes_{\mathbb{Z}} \mathbf{G}_{*}(B) \stackrel{\cong}{\rightrightarrows} \mathbf{G}_{*}^{G}(B)
$$


is an isomorphism (see [36, Lemma 5.6]), the lemma is equivalent to the assertion that the map

$$
\mathbf{G}_{0}^{G}(X) \otimes_{R(G)} \mathbf{G}_{*}^{G}(B) \rightarrow \mathbf{G}_{*}^{G}(B \times X)
$$

is an isomorphism.

Consider the cellular decomposition of $X$ as in Lemma 6.1. Then each $U_{i}=$ $X \backslash X_{i}$ is also a $T$-equivariantly cellular scheme. It suffices to show by induction on $i \geq 0$ that (6.7) holds when $X$ is any of these $U_{i}$ 's. There is nothing to prove for $i=0$ and the case $i=1$ follows by the homotopy invariance since $U_{1}$ is an affine space.

To prove the general case, we use the short exact sequence

$$
0 \rightarrow \mathbf{G}_{0}^{G}\left(U_{i+1} \backslash U_{i}\right) \rightarrow \mathbf{G}_{0}^{G}\left(U_{i+1}\right) \rightarrow \mathbf{G}_{0}^{G}\left(U_{i}\right) \rightarrow 0
$$

given by Lemma 6.1. This sequence splits, since each $\mathbf{G}_{0}^{G}\left(U_{i}\right)$ was shown to be free over $R(G)$ in Lemma 6.1. Tensoring this with $\mathbf{G}_{*}^{G}(B)$ over $R(G)$, we obtain a commutative diagram

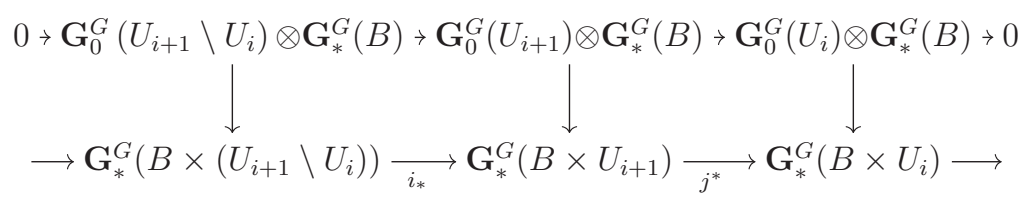

where the top row remains exact since the short exact sequence in (6.8) is split. The bottom row is the localization exact sequence. The left vertical arrow is an isomorphism by the homotopy invariance and the right vertical arrow is an isomorphism by the induction. In particular, $j^{*}$ is surjective in all indices. We conclude that $i_{*}$ is injective in all indices and the middle vertical arrow is an isomorphism.

Theorem 6.3 (Stacky Leray-Hirsch theorem). Suppose that $k$ is a perfect field and $B$ is a smooth scheme over $k$. Let $X$ be a $T$-equivariantly cellular scheme. Let $\mathfrak{F} \stackrel{i}{\rightarrow} \mathfrak{X} \stackrel{\pi}{\rightarrow} B$ be a Zariski locally trivial stack bundle (Section 6.1) each of whose fiber $\mathfrak{F}$ is a smooth stack of the form $[X / G]$. Assume that there are elements $\left\{e_{1}, \cdots, e_{r}\right\}$ in $\mathbf{K}_{0}(\mathfrak{X})$ such that $\left\{f_{1}=i^{*}\left(e_{1}\right), \cdots, f_{r}=i^{*}\left(e_{r}\right)\right\}$ is an $R(G)$-basis of $\mathbf{K}_{0}\left(\mathfrak{X}_{b}\right)$ for each fiber $\mathfrak{X}_{b}=\mathfrak{F}$ of the fibration. Then the map

$$
\begin{aligned}
& \Phi: \mathbf{K}_{*}^{G}(B) \underset{R(G)}{\otimes} \mathbf{K}_{0}(\mathfrak{F}) \rightarrow \mathbf{K}_{*}(\mathfrak{X}) \\
& \Phi\left(\sum_{1 \leq i \leq r} b_{i} \otimes f_{i}\right)=\sum_{1 \leq i \leq r} \pi^{*}\left(b_{i}\right) e_{i}
\end{aligned}
$$

is an isomorphism of $R(G)$-modules. In particular, $\mathbf{K}_{*}(\mathfrak{X})$ is a free $\mathbf{K}_{*}^{G}(B)$-module and the map $\pi^{*}: \mathbf{K}_{*}^{G}(B) \rightarrow \mathbf{K}_{*}(\mathfrak{X})$ is injective. 
Proof. Since $k$ is perfect and since the fibration $p$ is Zariski locally trivial, we can find a filtration

$$
\emptyset=B_{n+1} \subsetneq B_{n} \subsetneq \cdots \subsetneq B_{1} \subsetneq B_{0}=B
$$

of $B$ by closed subschemes such that for each $0 \leq i \leq n$, the scheme $B_{i} \backslash B_{i+1}$ is smooth and the given fibration is trivial over it. We set $U_{i}=B \backslash B_{i}$ and $V_{i}=$ $U_{i} \backslash U_{i-1}=B_{i-1} \backslash B_{i}$. Observe then that each of $U_{i}$ 's and $V_{i}$ 's is smooth.

Set $\mathfrak{X}_{i}=\pi^{-1}\left(U_{i}\right)$ and $\mathfrak{W}_{i}=\pi^{-1}\left(V_{i}\right)=V_{i} \times \mathfrak{F}$. Let $\eta_{i}: \mathfrak{X}_{i} \hookrightarrow \mathfrak{X}$ and $\iota_{i}: \mathfrak{W}_{i} \hookrightarrow \mathfrak{X}$ be the inclusion maps. We prove by induction on $i$ that the map $\mathbf{K}_{0}(\mathfrak{F}) \underset{R(G)}{\otimes} \mathbf{K}_{*}^{G}\left(U_{i}\right) \rightarrow \mathbf{K}_{*}\left(\mathfrak{X}_{i}\right)$ is an isomorphism, which will prove the theorem.

Since $U_{0}=\varnothing$ and $\mathfrak{X}_{1}=U_{1} \times \mathfrak{F}$, the desired isomorphism for $i \leq 1$ follows from Proposition 6.2 and the isomorphism $U_{1} \times \mathfrak{F} \simeq\left[\left(U_{1} \times X\right) / G\right]$. We now consider the commutative diagram:

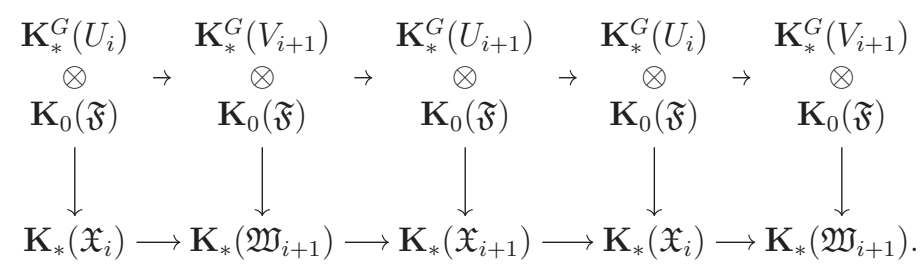

The top row in this diagram is obtained by tensoring the K-theory long exact localization sequence with $\mathbf{K}_{0}(\mathfrak{F})$ over $R(G)$, and the bottom row is just the localization exact sequence. Since $\mathbf{K}_{0}(\mathfrak{F})$ is a free $R(G)$-module ( $c f$. Lemma 6.1), the top row is also exact.

It is easily checked that the second and the third squares commute using the commutativity property of the push-forward and pull-back maps of K-theory of coherent sheaves in a Cartesian diagram of proper and flat maps. We show that the other squares also commute. It is enough to show that the first square commutes as the fourth one is same as the first. Let $\delta$ denote the connecting homomorphism in a long exact localization sequence for higher K-theory.

If we start with an element $b \otimes i^{*}\left(e_{j}\right) \in \mathbf{K}_{*}\left(U_{i}\right) \otimes \mathbf{K}_{0}(\mathfrak{F})$ and map this horizontally, we obtain $\delta b \otimes i^{*}\left(e_{j}\right)$ which maps vertically down to $\pi^{*}(\delta b) \cdot \iota_{i+1}^{*}\left(e_{j}\right)$. On the other hand, if we first map vertically, we obtain $\pi^{*}(b) \cdot \eta_{i}^{*}\left(e_{j}\right)$ which maps horizontally to $\delta\left(\pi^{*}(b) \cdot \eta_{i}^{*}\left(e_{j}\right)\right)$.

Now, we recall that these elements in the higher K-theory of coherent sheaves are represented by the elements in the higher homotopy groups of the various infinite loop spaces. Moreover, if we have a closed immersion of smooth stacks $\mathfrak{F} \hookrightarrow \mathfrak{X}$ with open complement $\mathfrak{U}$, then we have a fibration sequence of ring spectra

$$
\mathbf{K}(\mathfrak{F}) \rightarrow \mathbf{K}(\mathfrak{X}) \rightarrow \mathbf{K}(\mathfrak{U}) .
$$

The homotopy groups of these ring spectra form graded rings and the connecting homomorphism in the long exact sequence of the homotopy groups associated to 
the above fibration sequence satisfies the Leibniz rule (e.g., see [7, Appendix A] and [29, Section 2.4]).

Applying this Leibniz rule, we see that the term $\delta\left(\pi^{*}(b) \cdot \eta_{i}^{*}\left(e_{j}\right)\right)$ is same as $\delta \pi^{*}(b) \cdot \iota_{i+1}^{*}\left(e_{j}\right)=\pi^{*}(\delta b) \cdot \iota_{i+1}^{*}\left(e_{j}\right)$ since $\delta\left(\eta_{i}^{*}\left(e_{j}\right)\right)=0$. We have shown that the above diagram commutes.

The first and the fourth vertical arrows in (6.11) are isomorphisms by induction. The second and the fifth vertical arrows are isomorphisms by Proposition 6.2. Hence the middle vertical arrow is also an isomorphism by 5-lemma.

To show that $\pi^{*}$ is injective, consider the $T$-invariant filtration of $X$ as in (6.1) and let $j:\left[E\left(U_{1}\right) / G\right]=\mathfrak{X}_{1} \rightarrow \mathfrak{X}$ be the open inclusion. If we apply (6.9) to the map $\mathfrak{X}_{1} \rightarrow B$, we see that the composite map $\mathbf{K}_{*}^{G}(B) \rightarrow \mathbf{K}_{*}(\mathfrak{X}) \rightarrow \mathbf{K}_{*}\left(\mathfrak{X}_{1}\right)$ is an isomorphism (since $U_{1}$ is a $T$-invariant cell of $X$ ). We conclude that $\pi^{*}$ is split injective.

\section{Higher K-theory of toric stack bundles}

In this section, we give explicit descriptions of the higher K-theory of toric stack bundles in terms of the higher K-theory of the base scheme.

Let $T$ be a split torus of rank $n$. Let $N=\operatorname{Hom}\left(\mathbb{G}_{m}, T\right)$ be the lattice of oneparameter subgroups of $T$ and let $M=\operatorname{Hom}\left(T, \mathbb{G}_{m}\right)=N^{\vee}$ be its character group. Let $X=X(\Delta)$ be a smooth projective toric variety associated to a fan $\Delta$ in $N_{\mathbb{R}}$. Let

$$
0 \rightarrow G \rightarrow T \rightarrow T^{\prime} \rightarrow 0
$$

be an exact sequence of diagonalizable groups. This yields the exact sequence of the character groups

$$
0 \rightarrow T^{\prime \vee} \rightarrow T^{\vee} \rightarrow G^{\vee} \rightarrow 0
$$

\subsection{The Stanley-Reisner algebra associated to a subgroup of $T$}

We fix an ordering $\left\{\sigma_{1}, \cdots, \sigma_{m}\right\}$ of $\Delta_{\max }$ and let $\tau_{i} \subset \sigma_{i}$ be the cone which is the intersection of $\sigma_{i}$ with all those $\sigma_{j}$ such that $j \geq i$ and which intersect $\sigma_{i}$ in dimension $n-1$. Let $\tau_{i}^{\prime} \subset \sigma_{i}$ be the cone such that $\tau_{i} \cap \tau_{i}^{\prime}=\{0\}$ and $\operatorname{dim}\left(\tau_{i}\right)+$ $\operatorname{dim}\left(\tau_{i}^{\prime}\right)=n$ for $1 \leq i \leq m$. It is easy to see that $\tau_{i}^{\prime}$ is the intersection of $\sigma_{i}$ with all those $\sigma_{j}$ such that $j \leq i$ and which intersect $\sigma_{i}$ in dimension $n-1$. Since $X$ is smooth and projective, it is well known that we can choose the above ordering of $\Delta_{\max }$ such that

$$
\tau_{i} \subset \sigma_{j} \Rightarrow i \leq j \text { and } \tau_{i}^{\prime} \subset \sigma_{j} \Rightarrow j \leq i .
$$

Let $\Delta_{1}=\left\{\rho_{1}, \cdots, \rho_{d}\right\}$ be the set of one-dimensional cones in $\Delta$ and let $\left\{v_{1}, \cdots, v_{d}\right\}$ be the associated primitive elements of $N$. We choose $\left\{\rho_{1}, \cdots, \rho_{n}\right\}$ to be a set of one dimensional faces of $\sigma_{m}$ such that $\left\{v_{1}, \cdots, v_{n}\right\}$ is a basis of $N$. Let $\left\{\chi_{1}, \cdots, \chi_{n}\right\}$ be the dual basis of $M$. Let $\left\{\chi_{1}^{\prime}, \cdots, \chi_{r}^{\prime}\right\}$ be a chosen basis of $T^{\prime \vee}=M^{\prime}$. We will denote the group operations in all the lattices additively. 
Definition 7.1. Let $A$ be a commutative ring with unit and let $\left\{r_{1}, \cdots, r_{n}\right\}$ be a set of invertible elements in $A$. Let $I_{\Delta}^{T}$ denote the ideal of the Laurent polynomial algebra $A\left[t_{1}^{ \pm 1}, \cdots, t_{d}^{ \pm 1}\right]$ generated by the elements

$$
\left(t_{j_{1}}-1\right) \cdots\left(t_{j_{l}}-1\right), 1 \leq j_{p} \leq d
$$

such that $\rho_{j_{1}}, \cdots, \rho_{j_{l}}$ do not span a cone of $\Delta$. Let $J_{\Delta}^{G}$ denote the ideal of

$$
A\left[t_{1}^{ \pm 1}, \cdots, t_{d}^{ \pm 1}\right]
$$

generated by the relations

$$
s_{i}:=\left(\prod_{j=1}^{d}\left(t_{j}\right)^{<-\chi_{i}^{\prime}, v_{j}>}\right)-r_{i}, 1 \leq i \leq r .
$$

We define the $A$-algebras $R_{T}(A, \Delta)$ and $R_{G}(A, \Delta)$ to be quotients of $A\left[t_{1}^{ \pm 1}, \cdots, t_{d}^{ \pm 1}\right]$ by the ideals $I_{\Delta}^{T}$ and $I_{\Delta}^{G}=I_{\Delta}^{T}+J_{\Delta}^{G}$, respectively.

The ring $R_{G}(A, \Delta)$ will be called the Stanley-Reisner algebra over $A$ associated to the subgroup $G$. Every character $\chi \in M$ acts on $R_{T}(A, \Delta)$ via multiplication by the element $t_{\chi}=\left(\prod_{j=1}^{d}\left(t_{j}\right)^{<-\chi, v_{j}>}\right)$ and this makes $R_{T}(A, \Delta)$ (and hence $\left.R_{G}(A, \Delta)\right)$ an $(A \underset{\mathbb{Z}}{\otimes} R(T))$-algebra.

\subsection{The K-theory of toric stack bundles}

Let $T$ be a split torus over a perfect field $k$ and let $G$ be a closed subgroup of $T$ (which may not necessarily be a torus). Let $X$ be a smooth projective toric variety with dense torus $T$ and let $\pi: \mathfrak{X}=[(E(X) / G] \rightarrow B$ be a toric stack bundle over a smooth $k$-scheme $B$ associated to a principal $T$-bundle $p: E \rightarrow B$. We wish to describe the K-theory of $\mathfrak{X}$ in terms of the K-theory of $B$.

Any $T$-equivariant line bundle $L \rightarrow X$ uniquely defines a $G$-equivariant line bundle $E(L)=E \stackrel{T}{\times} L$ on $E(X)$, where the $G$-action on $E(L)$ is given exactly as on $E(X)$. Every $\rho \in \Delta_{1}$ defines a unique $T$-equivariant line bundle $L_{\rho}$ on $X$ with a $T$-equivariant section $s_{\rho}: X \rightarrow L_{\rho}$ which is transverse to the zero-section and whose zero locus is the orbit closure $V_{\rho}=\overline{O_{\rho}}$.

For any $\sigma \in \Delta$, let $u_{\sigma}$ denote the fundamental class $\left[\mathcal{O}_{V_{\sigma}}\right]$ of the $T$-invariant subscheme $V_{\sigma}$ in $\mathbf{K}_{0}^{T}(X)$ and let $y_{\sigma}$ denote the fundamental class of $\left[E\left(V_{\sigma}\right)\right]$ in $\mathbf{K}_{0}^{G}(E(X))=\mathbf{K}_{0}(\mathfrak{X})$.

Notice that $\bar{p}_{\sigma}: E\left(V_{\sigma}\right) \rightarrow B$ is a $G$-equivariant smooth projective toric subbundle of $\bar{p}: E(X) \rightarrow B$ with fiber $V_{\sigma}$. In particular, $\pi_{\sigma}:\left[E\left(V_{\sigma}\right) / G\right] \rightarrow B$ is a toric stack sub-bundle of $\pi: \mathfrak{X} \rightarrow B$ with fiber $\left[V_{\sigma} / G\right]$. We set $\mathfrak{X}_{\sigma}=$ $\left[E\left(V_{\sigma}\right) / G\right]$. 
Suppose that $\rho_{j_{1}}, \cdots, \rho_{j_{l}}$ do not span a cone in $\Delta$. Then $s=\left(s_{j_{1}}, \cdots, s_{j_{l}}\right)$ yields a $G$-equivariant nowhere vanishing section of $E\left(L_{\rho_{j_{1}}}\right) \oplus \cdots \oplus E\left(L_{\rho_{j_{l}}}\right)$ and hence the Whitney sum formula for Chern classes in K-theory implies that

$$
y_{\rho_{j_{1}}} \cdots y_{\rho_{j_{l}}}=0 \text { in } \mathbf{K}_{0}^{G}(E(X)) .
$$

We now consider the commutative diagram

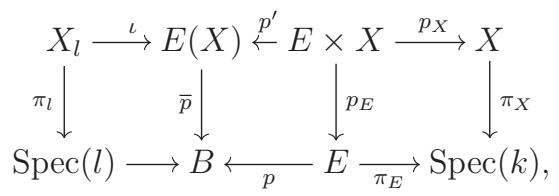

where $\operatorname{Spec}(l)$ is any point of $B$. It is clear that all squares are Cartesian and all the maps in the right square are $T$-equivariant.

We define $(T \times G)$-actions on any $T$-invariant subscheme $Y \subseteq X$ and on $E$ by $(t, g) \cdot y=t g \cdot y$ and $(t, g) \cdot e=t \cdot e$, respectively. An action of $(T \times G)$ on $E \times X$ is defined by $(t, g) \cdot(e, x)=(t \cdot e, t g \cdot x)$. It is clear that these are group actions such that the square on the right in $(7.7)$ is $(T \times G)$-equivariant. This implies that the middle square is also $(T \times G)$-equivariant and the map $\bar{p}$ is $G$-equivariant with respect to the trivial action of $G$ on $B$. The square on the left is $G$-equivariant.

Let $L_{\chi}$ denote the $T$-equivariant line bundle on $\operatorname{Spec}(k)$ associated to a character $\chi$ of $T$. Let $(T \times G)$ act on $L_{\chi}$ by $(t, g) \cdot v=\chi(t) \chi(g) \cdot v$. If $\chi \in M^{\prime}=T^{\prime \vee}$, then $G$ acts trivially on $L_{\chi}$ and hence it acts trivially on $\pi_{E}^{*}\left(L_{\chi}\right)$. Recall that $(T \times G)$ acts on $E$ via $T$. Hence $\pi_{E}^{*}\left(L_{\chi}\right) \rightarrow E$ is a $(T \times G)$-equivariant line bundle on which $G$-acts trivially. Since the $T$-equivariant line bundles on $E$ are same as ordinary line bundles on $B$, we find that for every $\chi \in M^{\prime}$, there is a unique ordinary line bundle $\zeta_{\chi}$ on $B$ such that $\pi_{E}^{*}\left(L_{\chi}\right)=p^{*}\left(\zeta_{\chi}\right)$.

Since $G$ acts trivially on $B$, there is a canonical ring homomorphism $c_{B}$ : $\mathbf{K}_{*}(B) \rightarrow \mathbf{K}_{*}^{G}(B)$ such that the composite $\mathbf{K}_{*}(B) \stackrel{c_{B}}{\rightarrow} \mathbf{K}_{*}^{G}(B) \rightarrow \mathbf{K}_{*}(B)$ is identity. These maps are simply the maps $\mathbf{K}_{*}(B) \stackrel{c_{B}}{\longrightarrow} \mathbf{K}_{*}^{G}(B)=\mathbf{K}_{*}(B) \otimes_{\mathbb{Z}} R(G) \rightarrow$ $\mathbf{K}_{*}(B)$. Since $p_{X}^{*} \circ \pi_{X}^{*}\left(L_{\chi}\right)=p_{E}^{*} \circ \pi_{E}^{*}\left(L_{\chi}\right)$ and since the $(T \times G)$-equivariant vector bundles on $E \times X$ are same as $G$-equivariant vector bundles on $E(X)$, we conclude that for every $\chi \in M^{\prime}$, there is a unique ordinary line bundle $\zeta_{\chi}$ on $B$ such that

$$
E\left(\pi_{X}^{*}\left(L_{\chi}\right)\right)=\bar{p}^{*}\left(\zeta_{\chi}\right)=\bar{p}^{*}\left(c_{B}\left(\zeta_{\chi}\right)\right) .
$$

Notice also that on each open subset of $B$ where the bundle $p$ is trivial, the restriction of $\zeta_{\chi}$ is the trivial line bundle since $\zeta_{\chi}$ is obtained from the $T$-line bundle $L_{\chi}$ on $\operatorname{Spec}(k)$.

We define a homomorphism of $\mathbf{K}_{*}(B)$-algebras $\mathbf{K}_{*}(B)\left[t_{1}^{ \pm 1}, \cdots, t_{d}^{ \pm 1}\right] \rightarrow \mathbf{K}_{*}(\mathfrak{X})$ by the assignment $t_{i} \mapsto\left[E\left(L_{\rho_{i}}^{\vee}\right) / G\right]$ for $1 \leq i \leq d$. If we let $r_{i}=\zeta_{\chi_{i}^{\prime}}$ for $1 \leq i \leq r$ (cf. Section 7.1), then it follows from (7.6) and (7.8) that this homomorphism descends to a $\mathbf{K}_{*}(B)$-algebra homomorphism

$$
\Phi_{G}: R_{G}\left(\mathbf{K}_{*}(B), \Delta\right) \rightarrow \mathbf{K}_{*}(\mathfrak{X}) .
$$


Given a sequence $\gamma=\left\{i_{1}, \cdots, i_{d}\right\}$ of integers, set

$$
E(\gamma)=E\left(\left(L_{\rho_{1}}^{\vee}\right)^{i_{1}} \otimes \cdots \otimes\left(L_{\rho_{d}}^{\vee}\right)^{i_{d}}\right) .
$$

We then see that for a monomial $\gamma(\underline{t})=t_{1}^{i_{1}} \cdots t_{d}^{i_{d}}$, we have

$$
\Phi_{G}(\gamma(\underline{t}))=[E(\gamma) / G] \text {. }
$$

The following result describes the higher K-theory of the toric stack bundle $\pi$ : $\mathfrak{X} \rightarrow B$.

Theorem 7.2. The homomorphism $\Phi_{G}$ is an isomorphism.

Before we prove this theorem, we consider some special cases which will be used in the final proof. The following observations will be used throughout the proofs.

The first observation is that the cell closures of $X$ are the $T$-equivariant subschemes $V_{\tau_{i}}$. So the classes of $\mathcal{O}_{V_{\tau_{i}}}$ form an $R(G)$-basis of $\mathbf{K}_{0}^{G}(X)$ by Lemma 6.1. Since $\iota^{*}\left(y_{\tau_{i}}\right)=\left[\mathcal{O}_{V_{\tau_{i}}}\right]$, we see that Theorem 6.3 applies to the toric stack bundle $\pi: \mathfrak{X} \rightarrow B$.

Second observation is that $G$ is a diagonalizable group which acts trivially on $B$. Hence the map $\mathbf{K}_{*}(B) \otimes R(G) \rightarrow \mathbf{K}_{*}^{G}(B)$ is a ring isomorphism by [36, Lemma 5.6]. This identification will be used without further mention. Since any character $\chi \in M$ acts on $R_{T}\left(\mathbf{K}_{*}(B), \Delta\right)$ and $\mathbf{K}_{*}^{G}(E(X))$ via multiplication by $t_{\chi}$ and $\Phi_{G}\left(t_{\chi}\right)$ respectively ( $c f$. [32, Proposition 4.3]), we observe that the composite $\operatorname{map} R_{T}\left(\mathbf{K}_{*}(B), \Delta\right) \rightarrow R_{G}\left(\mathbf{K}_{*}(B), \Delta\right) \rightarrow \mathbf{K}_{*}^{G}(E(X))$ is $\mathbf{K}_{*}^{T}(B)$-linear.

Remark 7.3. We remark that the result of Thomason in [36, Lemma 5.6] is stated for affine schemes, but his proof works for all schemes. Another way to deduce the general case from the affine case is to get a stratification of $B$ by affine subschemes as in (6.10), use induction on the number of affine strata, the localization sequence and the fact that $R(G)$ is free over $\mathbb{Z}$.

Lemma 7.4. The homomorphism $\Phi_{G}$ is an isomorphism when $G=T$.

Proof. In this case, we first notice that the map $R_{T}(\mathbb{Z}, \Delta) \stackrel{\phi}{\rightarrow} \mathbf{K}_{0}^{T}(X)$ which takes $t_{i}$ to $\left[L_{\rho_{i}}^{\vee}\right]$, is an isomorphism of $R(T)$-algebras by [41, Theorem 6.4]. On the other hand, we have the maps

$$
\mathbf{K}_{*}(B) \underset{\mathbb{Z}}{\otimes} R_{T}(\mathbb{Z}, \Delta) \stackrel{\simeq}{\rightarrow} R_{T}\left(\mathbf{K}_{*}(B), \Delta\right) \stackrel{\Phi_{T}}{\longrightarrow} \mathbf{K}_{*}^{T}(E(X)),
$$

where the first map takes $\alpha \otimes t_{i}$ to $\alpha \cdot t_{i}$ for $1 \leq i \leq d$. This map is clearly an isomorphism (see (7.4)). It is clear from the definition of $\Phi_{T}$ that the composite map is the same as the map $\Phi$ in (6.9) (with $G=T$ ). It follows from Theorem 6.3 that the composite map in (7.11) is an isomorphism. We conclude that $\Phi_{T}$ is an isomorphism. 
Corollary 7.5. For any closed subgroup $G \subseteq T$, the ring $R_{G}\left(\mathbf{K}_{*}(B), \Delta\right)$ is a free $\mathbf{K}_{*}(B)$-module.

Proof. We have seen above that the image of a character $\chi \in M$ in $R_{T}\left(\mathbf{K}_{*}(B), \Delta\right)$ is $t_{\chi}$. If we let $J^{G}$ denote the ideal $\left(\chi_{1}^{\prime}-\zeta_{\chi_{1}^{\prime}}, \cdots, \chi_{r}^{\prime}-\zeta_{\chi_{r}^{\prime}}\right)$ in $\mathbf{K}_{*}^{T}(B)$, then it follows from (7.5) that $J_{\Delta}^{G}=J^{G} R_{T}\left(\mathbf{K}_{*}(B), \Delta\right)$ under the $\operatorname{map} \mathbf{K}_{*}^{T}(B) \rightarrow R_{T}\left(\mathbf{K}_{*}(B), \Delta\right)$.

It follows from Lemma 7.4 and Theorem 6.3 (with $G=T$ ) that $R_{T}\left(\mathbf{K}_{*}(B), \Delta\right.$ ) is a free $\mathbf{K}_{*}^{T}(B)$-module. This implies that $R_{G}\left(\mathbf{K}_{*}(B), \Delta\right)=R_{T}\left(\mathbf{K}_{*}(B), \Delta\right) / J_{\Delta}^{G}$ is a free $\mathbf{K}_{*}^{T}(B) / J^{G}$-module. Thus, it suffices to show that $\mathbf{K}_{*}^{T}(B) / J^{G}$ is a free $\mathbf{K}_{*}(B)$-module. Since $\mathbf{K}_{*}^{T}(B)$ is isomorphic to a Laurent polynomial ring $\mathbf{K}_{*}(B)\left[x_{1}^{ \pm 1}, \cdots, x_{n}^{ \pm 1}\right]$ and since each character $\chi \in M^{\prime}$ is a monomial in this ring, the desired freeness follows from Lemma 7.7.

Lemma 7.6. The homomorphism $\Phi_{G}$ is an isomorphism when $p: E \rightarrow B$ is a trivial principal bundle.

Proof. Since $p: E \rightarrow B$ is a trivial bundle, we have observed before that $\zeta_{\chi_{i}^{\prime}}=1$ for each $1 \leq i \leq r$. In particular, the $\operatorname{map} \mathbf{K}_{*}^{T}(B) / J^{G} \rightarrow \mathbf{K}_{*}^{G}(B)$ is an isomorphism by Lemma 7.8, where $J^{G}$ is as in Corollary 7.5.

It follows from Theorem 6.3 and Lemma 7.4 that $\Phi_{T}$ is an isomorphism of free $\mathbf{K}_{*}^{T}(B)$-modules. This implies that $R_{G}\left(\mathbf{K}_{*}(B), \Delta\right)=R_{T}\left(\mathbf{K}_{*}(B), \Delta\right) / J_{\Delta}^{G}$ is a free $\mathbf{K}_{*}^{T}(B) / J^{G}=\mathbf{K}_{*}^{G}(B)$-module. It follows from this and Theorem 6.3 that $\Phi_{G}$ is a basis preserving homomorphism of free $\mathbf{K}_{*}^{G}(B)$-modules of same rank. Hence, it must be an isomorphism.

Lemma 7.7. Let $S=A\left[x_{1}^{ \pm 1}, \cdots, x_{n}^{ \pm 1}\right]$ be a Laurent polynomial ring over a commutative ring $A$ with unit. Let $\left\{t_{1}, \cdots, t_{r}\right\}$ be a set of monomials in $S$ and let $\left\{u_{1}, \cdots, u_{r}\right\}$ be a set of units in $A$. Then the ring $\frac{S}{\left(t_{1}-u_{1}, \cdots, t_{r}-u_{r}\right)}$ is free over $A$.

Proof. This is left as an easy exercise using the fact that $S$ is a free $A$-module on the monomials.

Lemma 7.8. Let $A$ be a commutative ring with unit and let

$$
0 \rightarrow L \rightarrow M \rightarrow N \rightarrow 0
$$

be a short exact sequence of finitely generated abelian groups. Let $I_{L}$ be the ideal of the group ring $A[M]$ generated by the set $\{s-1 \mid s \in S\}$, where $S$ is a generating set of $L$. Then the map of group rings $\frac{A[M]}{I_{L}} \rightarrow A[N]$ is an isomorphism.

Proof. This is an elementary exercise and a proof can be found in [26, Proposition 2].

Proof of Theorem 7.2. We shall prove this theorem along the same lines as the proof of Theorem 6.3. Recall that our base field $k$ is perfect. We consider the 
stratification of $B$ by smooth locally closed subschemes as in (6.10). We shall follow the notations used in the proof of Theorem 6.3. It suffices to show by induction on $i$ that the theorem is true when $B$ is replaced by each $U_{i}$. Since $U_{0}=\emptyset$ and since $E \stackrel{p}{\rightarrow} B$ is trivial over $U_{1}$, the desired isomorphism for $i \leq 1$ follows from Lemma 7.6.

Given a smooth locally closed subscheme $j: U \hookrightarrow B$, let $\zeta_{i}^{U}=j^{*}\left(\zeta_{\chi_{i}^{\prime}}\right) \in$ $\mathbf{K}_{*}(U)$ for $1 \leq i \leq r$ and set $J_{U}^{G}=\left(\chi_{1}^{\prime}-\zeta_{1}^{U}, \cdots, \chi_{r}^{\prime}-\zeta_{r}^{U}\right)$.

We have seen in the proof of Lemma 7.4 that for any such inclusion $U \subseteq B$, $R_{T}\left(\mathbf{K}_{*}(U), \Delta\right)$ is same as $\mathbf{K}_{0}^{T}(X) \underset{\mathbb{Z}}{\otimes \mathbf{K}_{*}}(U)$. Moreover, the maps

$$
\begin{aligned}
& R_{G}\left(\mathbf{K}_{*}(B), \Delta\right) \underset{\mathbf{K}_{*}(B)}{\otimes} \mathbf{K}_{*}(U) \\
& \simeq \frac{R_{T}\left(\mathbf{K}_{*}(B), \Delta\right)}{J^{G} R_{T}\left(\mathbf{K}_{*}(B), \Delta\right)} \underset{\mathbf{K}_{*}(B)}{\otimes} \mathbf{K}_{*}(U) \rightarrow \frac{R_{T}\left(\mathbf{K}_{*}(U), \Delta\right)}{J_{U}^{G} R_{T}\left(\mathbf{K}_{*}(U), \Delta\right)} \\
& \rightarrow R_{G}\left(\mathbf{K}_{*}(U), \Delta\right)
\end{aligned}
$$

are all isomorphisms.

We now consider the diagram:

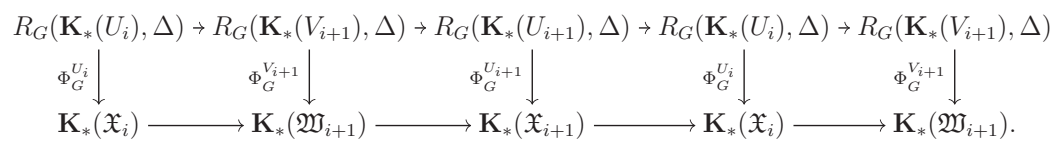

Using (7.12), we see that the top row of (7.13) is obtained by tensoring the localization exact sequence

$$
\cdots \rightarrow \mathbf{K}_{*}\left(U_{i}\right) \rightarrow \mathbf{K}_{*}\left(V_{i+1}\right) \rightarrow \mathbf{K}_{*}\left(U_{i+1}\right) \rightarrow \mathbf{K}_{*}\left(U_{i}\right) \rightarrow \mathbf{K}_{*}\left(V_{i+1}\right) \rightarrow \cdots
$$

of $\mathbf{K}_{*}(B)$-modules with $R_{G}\left(\mathbf{K}_{*}(B), \Delta\right)$. Hence, this row is exact by Corollary 7.5. The bottom row is anyway a localization exact sequence.

We now show that the diagram (7.13) commutes. It is clear that the third square commutes and the fourth square is same as the first. So we need to check that the first two squares commute.

Let $\alpha: V_{i+1} \hookrightarrow U_{i+1}$ and $\beta: \mathfrak{M}_{i+1} \hookrightarrow \mathfrak{X}_{i+1}$ be the closed immersions of smooth schemes and stacks. Following the notations in the proof of Theorem 6.3, we see that for any $u \in \mathbf{K}_{*}\left(U_{i}\right)$ and for any monomial $\gamma(\underline{t})=t_{1}^{i_{1}} \cdots t_{d}^{i_{d}}$,

$$
\begin{aligned}
\delta \circ \Phi_{G}^{U_{i}}(u \otimes \gamma(\underline{t})) & =\delta\left(\pi_{U_{i}}^{*}(u) \cdot \eta_{i}^{*}([E(\gamma) / G])\right) \\
& =\delta\left(\pi_{U_{i}}^{*}(u)\right) \cdot \iota_{i+1}^{*}([E(\gamma) / G]) \\
& =\pi_{V_{i+1}^{*}}^{*}(\delta(u)) \cdot \iota_{i+1}^{*}([E(\gamma) / G]) \\
& =\Phi_{G}^{V_{i+1}}(\delta(u) \otimes \gamma(\underline{t})) \\
& =\Phi_{G}^{V_{i+1}} \circ \delta(u \otimes \gamma(\underline{t}))
\end{aligned}
$$


where $E(\gamma) \in \mathbf{K}_{*}(\mathfrak{X})$ is as in (7.10). The second equality follows from the Leibniz rule and the third equality follows from the commutativity of (6.11). This shows that the first (and the last) square commutes.

To show the commutativity of the second square, let $v \in \mathbf{K}_{*}\left(V_{i+1}\right)$. We then have

$$
\begin{aligned}
\beta_{*} \circ \Phi_{G}^{V_{i+1}}(v \otimes \gamma(\underline{t})) & =\beta_{*}\left(\pi_{V_{i+1}}^{*}(v) \cdot \iota_{i+1}^{*}([E(\gamma) / G])\right) \\
& =\beta_{*}\left(\pi_{V_{i+1}}^{*}(v) \cdot \beta^{*} \circ \eta_{i+1}^{*}([E(\gamma) / G])\right) \\
& =\beta_{*}\left(\pi_{V_{i+1}}^{*}(v)\right) \cdot \eta_{i+1}^{*}([E(\gamma) / G]) \\
& =\pi_{U_{i+1}}^{*}\left(\alpha_{*}(v)\right) \cdot \eta_{i+1}^{*}([E(\gamma) / G]) \\
& =\Phi_{G+1}^{U_{i+1}}\left(\alpha_{*}(v) \otimes \gamma(\underline{t})\right) \\
& =\Phi_{G}^{U} \circ \alpha_{*}(v \otimes \gamma(\underline{t})),
\end{aligned}
$$

where third equality follows from the projection formula and the fourth equality follows from the commutativity of (6.11). This shows that the second square commutes.

The first and the fourth vertical arrows are isomorphisms by induction. The second and the fifth vertical arrows are isomorphisms by Lemma 7.6. Hence the middle vertical arrow is also an isomorphism by 5-lemma. This concludes the proof of Theorem 7.2.

Remark 7.9. It was assumed in Theorem 7.2 that $G$ is a subgroup of $T$. Since $\mathfrak{X}$ is just the toric stack $[E(X) / G]$ associated to the data $(E(X), G \stackrel{\phi}{\rightarrow} T)$, the general case can always be reduced to the case of Theorem 7.2. We refer to Remark 4.5 for how this can be done.

\section{References}

[1] S. Baggio, Equivariant K-theory of smooth toric varieties, Tohoku Math. J. (2) 59 (2007), 203-231.

[2] A. Bialynicki-Birula, Some theorems on actions of algebraic groups, Ann. of Math. (2) 98 (1973), 480-497.

[3] A. Borel, "Linear Algebraic Groups", Graduate Text in Mathematics, Vol. 126, $2^{\text {nd }}$ edition, Springer-Verlag, 1991.

[4] L. BORISOV and P. HORJA, On the K-theory of smooth Deligne-Mumford stacks, In: "Snowbird Lectures on String Geometry", Contemp. Math., Vol. 401, Amer. Math. Soc., Providence, RI, 2006, 21-42.

[5] M. BRION, Private communication to the authors, 2012.

[6] M. BRION and D. LUNA, Sur la structure locale de variétés sphériques, Bull. Soc. Math. France 115 (1987), 211-226.

[7] K. BRown, Abstract homotopy and generalized sheaf cohomology, Trans. Amer. Math. Soc. 186 (1973), 419-458.

[8] D. Cox, J. LitTle and H. SCHEnck, "Toric Varieties", Graduate Studies in Mathematics, Vol. 124, Amer. Math. Soc., Providence, 2011. 
[9] D. Edidin and W. Graham, Equivariant intersection theory, Invent. Math. 131 (1998), 595-634.

[10] A. Elmendorf, I. Kriz, M. Mandell and P. May, "Rings, Modules, and Algebras in Stable Homotopy Theory", Mathematical Surveys and Monographs, with an appendix by M. Cole, Vol. 47, American Mathematical Society, Providence, RI, 1997.

[11] B. FAnTEChi, E. Mann and F. Nironi, Smooth toric DM stacks, J. Reine Angew. Math. 648 (2010), 201-244.

[12] W. Fulton, "Introduction to Toric Varieties", Annals of Mathematics Studies, Vol. 131, Princeton University Press, Princeton, NJ, 1993.

[13] R. Goldin, M. Harada, T. Holm and T. Kimura, The full orbifold $K$-theory of Abelian symplectic quotients, J. $K$-theory 8 (2011), 339-362.

[14] A. Gerashenko and M. Satriano, Toric stacks I: The theory of stacky fans, Trans. Amer. Math. Soc. 367 (2015), 1033-1071.

[15] A. GERASHENKO and M. SATRIANO, Toric stacks II: Intrinsic characterization of toric stacks, Trans. Amer. Math. Soc. 367 (2015), 1073-1094.

[16] A. Grothendieck and J. Dieudonné, Elements de Géométrie Algébrique IV, Étude locale des schémas et des morphismes de schémas, Publ. Math. Inst. Hautes Étud. Sci. 28 (1966), 1-255.

[17] Z. HUA, On the Grothendieck groups of toric stacks, arXiv:0904.2824v1, (2009).

[18] W. HeSSELINK, Concentration under actions of algebraic groups, In: "Malliavin Algebra Seminar", Paul Dubreil and Marie-Paule, 33 ${ }^{\text {rd }}$ Year (Paris), Lecture Notes Math., Vol. 867, Springer-Verlag, Berlin, 1980, 55-89.

[19] Y. JiAng and H. TSEnG, On the K-theory of toric stack bundles, Asian J. Math. 14 (2010), $1-10$.

[20] R. JoshuA, Algebraic K-theory and higher Chow groups of Linear varieties, Math. Proc. Cambridge. Philos. Soc. 130 (2001), 37-60.

[21] R. JoshUA and A. KRISHNA, Motivic cohomology of toric stacks, 2012, in preparation.

[22] A. KRISHNA, Riemann-Roch for equivariant higher K-theory, Adv. Math. 262 (2014), 126192.

[23] A. KRISHNA, Higher Chow groups of varieties with group actions, Algebra Number Theory 7 (2013), 449-506.

[24] M. LEVINE, Algebraic K-theory of the classical groups and some twisted forms, Duke Math. J. 70 (1993), 405-443.

[25] A. MASSEY, The KH-theory of complete simplicial toric varieties and the algebraic $K$ theory of weighted projective spaces, J. Pure Appl. Algebra 217 (2013), 2108-2116.

[26] W. MAY, Commutative group algebras, Trans. Amer. Math. Soc. 136 (1969), 139-149.

[27] A. MerKuRJev, Comparison of the equivariant and the standard K-theory of algebraic varieties, Algebra i Analiz 9 (1997), 175-214; St. Petersburg Math. J. 9 (1998), 815-850.

[28] R. MorelLI, The K-theory of a toric variety, Adv. Math. 100 (1993), 154-182.

[29] I. PANIn, Oriented cohomology theories of algebraic varieties, $K$-Theory 30 (2003), 265314.

[30] F. Perroni, A note on toric Deligne-Mumford stacks, Tohoku Math. J. (2) 60 (2008), 441458.

[31] D. Quillen, Higher Algebraic K-theory: I, Lecture Notes in Mathematics, Vol. 341, Springer-Verlag, Berlin, 1971, 85-147.

[32] P. Sankaran and V. Uma, Cohomology of toric bundles, Comment. Math. Helv. 78 (2003), 540-554.

[33] B. SHIPLEY and S. SCHWEDE, Algebras and modules in monoidal model categories, Proc. Lond. Math. Soc. (3) 80 (2000), 491-511.

[34] R. Steinberg, On a theorem of Pittie, Topology 14 (1975), 173-177.

[35] Y. TAKEDA, On the K-groups of spherical varieties, Osaka J. Math. 35 (1998), 73-81.

[36] R. ThOmASOn, Lefschetz Riemann-Roch theorem and coherent trace formula, Invent. Math. 85 (1986), 515-543. 
[37] R. Thomason, Comparison of equivariant algebraic and topological K-theory, Duke Math. J. 53 (1986), 795-825.

[38] R. THOMASON, Algebraic K-theory of group scheme actions, In: "Algebraic Topology and Algebraic $K$-theory", Ann. of Math. Studies, Vol. 113, 1987, 539-563.

[39] R. Thomason, Equivariant algebraic vs. topological K-homology Atiyah-Segal-style, Duke Math. J. 56 (1988), 589-636.

[40] B. Totaro, Chow groups, Chow cohomology, and linear varieties, Forum Math. Sigma 2, Article ID e17, 25 p., electronic only (2014).

[41] G. Vezzosi and A. VISTOLI, Higher algebraic K-theory for actions of diagonalizable groups, Invent. Math. 153 (2003), 1-44.

[42] A. VISTOLI, Higher equivariant K-theory for finite group actions, Duke Math. J. 63 (1991), 399-419.

Department of Mathematics

Ohio State University

Columbus, Ohio

43210, USA

joshua@math.ohio-state.edu

School of Mathematics

Tata Institute of Fundamental Research

Homi Bhabha Road, Colaba

Mumbai, India

amal@math.tifr.res.in 